\title{
Un nouvel indice de la productivité agricole : les baux de Notre-
}

\section{Dame de Paris, 1450-1789}

Philip T. Hoffman

\section{Résumé}

Philip T. Hoffman. Un nouvel indice de la productivité agricole : les baux de Notre-Dame de Paris, 1450-1789. Cet article présente une nouvelle méthode pour mesurer la productivité agricole pour la période précédant les recensements agraires. Cette méthode repose sur les données concernant les prix et les taux de fermage afin de calculer la productivité totale des facteurs (PTF). La méthode est à la fois plus riche d'informations et plus fiable que la comparaison habituelle entre les récoltes, les rendements et le produit

par travailleur ; cet article l'explique en utilisant un échantillon de contrats de fermage tirés des archives de Notre-Dame de Paris. L'analyse de l'échantillon révèle quelques-unes des causes de la croissance agricole sous l'Ancien Régime et suggère que l'agriculture de cette période était capable d'un développement considérable du moins dans le Bassin parisien. Cependant, la croissance observée était extrêmement sensible aux crises politiques.

\begin{abstract}
Philip T. Hoffman A New Index of Agricultural Productivity : Les baux de Notre-Dame de Paris, 1450-1789.

This paper presents a new method of measuring agricultural productivity in the era before agricultural censuses, a method that relies on evidence concerning prices and land rental rates to calculate the total factor productivity of agriculture. The method is both more informative and more reliable than the typical comparisons of crop yields and output per worker, and the paper explains it using a sample of leases drawn from the archives of the Cathedral of Notre Dame. The analysis of this sample reveals some of the causes of agricultural growth under the Old Regime and suggests that the agriculture of the période was capable of considerable development, at least in the Paris Basin. What growth occurred, though, was extremely sensitive to political crises.
\end{abstract}

\section{Citer ce document / Cite this document :}

Hoffman Philip T. Un nouvel indice de la productivité agricole : les baux de Notre-Dame de Paris, 1450-1789. In: Histoire \& Mesure, 1991 volume $6-n^{\circ} 3-4$. Varia. pp. 215-243.

doi : 10.3406/hism.1991.1394

http://www.persee.fr/doc/hism_0982-1783_1991_num_6_3_1394

Document généré le 16/10/2015 
Histoire \& Mesure, 1991, VI-3/4, 215-243

\section{Philip T. HOFFMAN}

\section{Un nouvel indice de la productivité agricole : les baux de Notre-Dame de Paris, 1450-1789 (1)}

Ecrits sur un parchemin ou griffonnés sur un papier, exprimés en livres de comptes, les baux agricoles abondent dans les archives de l'Ancien Régime, aussi bien dans les études notariales que dans les archives familiales, hospitalières ou de maisons religieuses. Aussi, n'est-il guère surprenant que bon nombre d'historiens entreprenants en aient tiré profit (2). La majorité, cependant, s'est contentée de les utiliser pour étudier les revenus des propriétaires terriens ou pour estimer le poids des prélèvements sur la paysannerie. Les rares qui ont essayé d'en déduire un indice de la production agricole l'ont toujours fait presque en s'excusant tant l'emploi des dîmes semble en ce domaine s'imposer.

Les historiens ne se sont pas rendu compte que les baux peuvent éclairer d'un jour nouveau le problème de la productivité agricole. En effet, sous des conditions appropriées, leurs enseignements peuvent être combinés avec les prix des produits et les coûts des facteurs de production pour donner une mesure de la productivité. Celle-ci n'est pas entendue seulement comme la productivité de la terre ou du travail mais comme la productivité globale, définie par le rapport entre les outputs et les inputs calculés à partir de tous les facteurs de production utilisés (terre, travail et capital). Ce type d'utilisation des baux agricoles a déjà permis d'estimer la productivité de l'agriculture anglaise ancienne. Il manque, certes, une certaine rigueur statistique à ces études mais elles ont tout de même fourni des estimations plus solides que les anciennes données mal assurées de rendements par surface ou par travailleur. Or, il s'agit-là de résultats essentiels car ils permettent de déterminer si l'agriculture de l'Ancien Régime est soumise à de strictes rigidités techniques ou organisationnelles ou si, au contraire, certaines fermes peuvent produire plus avec les mêmes quantités de terre, de travail et de capital. En d'autres termes, c'est la possibilité d'une croissance économique ancienne qui peut être explorée.

Cette étude analyse 809 baux provenant des archives de NotreDame de Paris pour une période qui s'étend de 1450 à 1789. Ces baux forment 39 séries qui chacune concerne une propriété particulière, localisée dans l'un des 25 villages de la région parisienne de la figure 1 . Ces propriétés sont en moyenne situées à moins de 40 kilomètres de Paris, la plus proche à environ cinq kilomètres et la plus éloignée à 


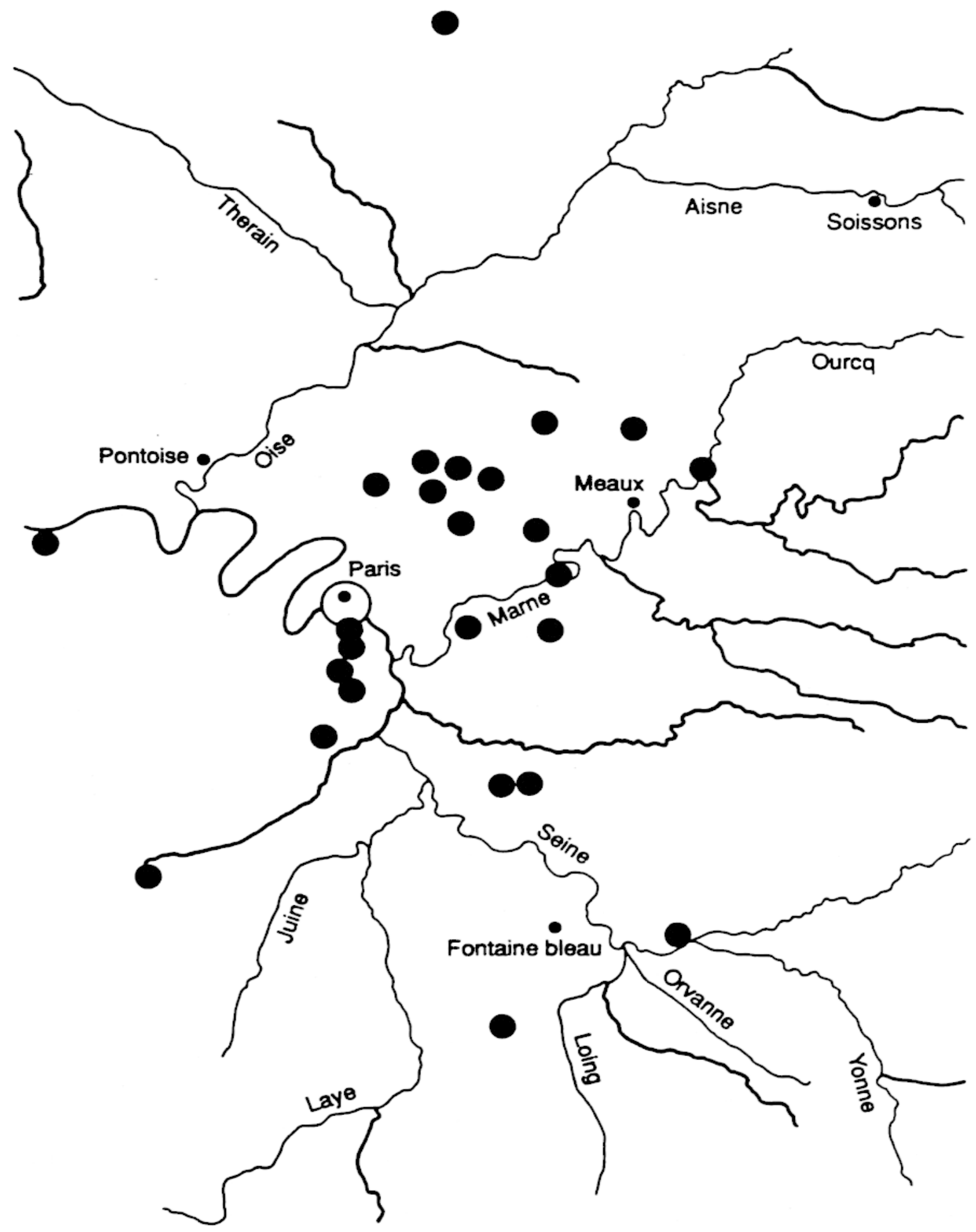

Figure 1 : Carte de localisation des propriétés utilisées.

96 kilomètres. La plupart sont louées avec quelques droits mineurs sur la collecte de dîmes locales ou de droits seigneuriaux. Presqu'aucune ne change de dimension de manière significative ; dans le cas contraire, une 
autre série est commencée. Les superficies sont très inégales, s'étageant de 0,26 hectare à 278 hectares, avec une moyenne de 67 hectares. Elles sont consacrées de manière prépondérante à la céréaliculture : seulement $1,4 \%$ des terres sont en vignes et $4,8 \%$ en prés (3).

L'échantillon n'est pas aléatoire - peu de propriétés sont situées à l'ouest de Paris - mais il semble représentatif de l'agriculture d'openfield dans la périphérie urbaine. Il nous permet, de plus, de suivre dans le long terme un grand nombre de propriétés inchangées. Il y a ainsi peu de risque que le mouvement observé de la productivité soit un simple artefact statistique résultant d'un changement dans la composition de l'échantillon. Un saut de la courbe au XVIII ${ }^{\mathbf{e}}$ siècle, par exemple, n'est probablement pas qu'un accident consécutif à l'inclusion de propriétés situées sur de meilleurs sols.

Cet échantillon offre un dernier avantage: nous connaissons les caractéristiques de chacune des propriétés (superficie, localisation, nature des récoltes, identité du tenancier...). Aussi est-il envisageable d'interpréter les variations de la productivité avec plus de précision qu'auparavant. Le résultat final donne le tableau saisissant d'une agriculture capable d'une croissance considérable, au moins dans les environs de Paris, et dont il est désormais possible de discerner les facteurs de développement et de stagnation.

\section{COMMENT UTILISER LES BAUX ET LES PRIX POUR MESURER LA PRODUCTIVITÉ?}

Aujourd'hui, il est facile d'estimer la productivité du travail agricole en divisant les quantités produites (hectolitres de blé, par exemple) par le nombre de travailleurs employés. Pour l'Ancien Régime, la même opération est sans espoir; les quelques tentatives en ce sens n'ont abouti qu'à des résultats douteux. La difficulté est de déterminer la quantité de travail utilisée, constatation souvent délicate à établir avec certitude même avec les données du XIX ${ }^{\mathrm{c}}$ siècle. Comment estimer la fraction de la population rurale qui travaille dans les fermes quand tant d'habitants de la campagne se consacrent à des activités artisanales (4) ? Il est tout aussi difficile de retracer l'évolution de la productivité de la terre. La voie la plus sûre serait de calculer les rendements en grains à partir d'un ensemble varié de sources et d'en déduire la productivité de la terre. La documentation est cependant toujours trop insuffisante pour ne pas faire de ces comparaisons une entreprise hasardeuse. Les rendements en grains par hectare peuvent considérablement varier d'un bout à l'autre d'une ferme ou d'un village à l'autre, jetant le doute sur une comparaison, par exemple, entre les rendements pour un champ particulier tirés d'un inventaire du XVI ${ }^{c}$ siècle, et ceux obtenus à partir d'une enquête du $\mathrm{XIX}^{\mathrm{e}}$ siècle sur l'ensemble d'un arrondissement. De manière générale, les variations temporelles rendent problématique toute comparaison interséculaire. Pire, même des moyennes en apparence crédibles peuvent se révéler fausses. Si, par exemple, la culture du blé s'étend au détriment de celle de céréales de moindre valeur sur des sols de médiocre qualité, 
les rendements moyens pourront diminuer même si, dans le même temps, le produit physique par hectare de terrain à qualité constante augmente (5).

Par ailleurs, l'emploi des dîmes pour estimer la productivité de la terre se heurte à un sérieux problème: les séries que les historiens s'efforcent de constituer omettent de prendre en compte les innovations comme les prairies artificielles ou les nouvelles récoltes comme les navets. Un profil plat d'une courbe de dîme peut donc masquer une productivité en augmentation (6).

Il est donc difficile de procéder à des estimations fiables pour l'Ancien Régime de la productivité du travail, des rendements physiques ou de toute autre mesure de la productivité de la terre. Qui plus est, aucune de ces approches ne nous donne la productivité totale des facteurs: même quand elles semblent assez bien fondées, elles ne fournissent que des productivités partielles soit de la terre, soit du travail, et le plus souvent seulement pour un seul produit, en général le blé. Rien n'est dit sur le capital agricole et les autres facteurs de production. Rien non plus à propos des productions que les dîmes n'intègrent pas, comme la laine ou le bétail, qui ne sont pourtant pas négligeables même dans les régions de céréaliculture. Un nouveau type d'informations apparaît évidemment nécessaire, à partir desquelles il serait possible de mesurer la productivité non pas d'un seul facteur mais la productivité globale.

C'est précisément ce que l'usage des baux permet d'envisager. Combinées avec les prix et les salaires, les données sur la rente foncière autorisent une mesure de la productivité totale des facteurs. Bien qu'elle n'échappe pas à certaines critiques, elle semble beaucoup plus digne de confiance que les douteuses mesures physiques par travailleur ou par hectare. Cet emploi des données de prix et de rentes a été suggéré pour la première fois par Donald McCloskey dans une étude sur les enclosures anglaises. Plus récemment, Robert Allen a utilisé avec succès la même méthode pour examiner la productivité des enclosures et de l'agriculture anglaise en général au XVIII' siècle (7).

McCloskey et Allen s'appuient sur le fait que la productivité totale des facteurs (PTF) peut être calculée à partir des prix et des baux de rentes en lieu et place des mesures physiques réelles des productions et des facteurs de production. La définition de la PTF est classique : elle estime l'efficience des produits de l'entreprise agricole et peut être grossièrement définie comme la production moyenne de tous les intrants utilisés. Son taux de croissance est égal à celui de la production globale moins celui de l'emploi des facteurs de production, chaque produit étant pondéré par sa part dans le revenu total et chaque facteur par sa part dans le coût total. $\mathrm{Si}$, de plus, nous supposons, comme R. Allen, que ces proportions sont constantes (hypothèse raisonnable pour l'agriculture de l'Ancien Régime), alors la PTF est proportionnelle à $(\mathbf{r}+\mathbf{t})^{\mathbf{s}} \mathbf{C} / \mathbf{P}$, où $r$ est la rente par hectare, $t$ les prélèvements fiscaux par hectare, $\mathbf{s}$ la part de la terre dans les facteurs de production, $\mathbf{C}$ un indice géométrique des coûts des autres facteurs pondérés par leur part dans le coût total et $P$ un indice géométrique des prix des productions agricoles pondérés par leur part respective dans le revenu total. En termes non mathématiques, 
la PTF est forte si l'exploitation peut supporter des impôts et une rente élevés en dépit du coût important des autres facteurs de production et des bas prix des produits.

En résumé, le calcul de cet indice exige de connaître les proportions entres les productions d'une part et entre les facteurs de production d'autre part, les prix des produits agricoles, et le coût des différents facteurs de production (dont la terre, son prix étant simplement mesuré par la rente et les impôts qu'elle supporte). Il est alors possible de mesurer la PTF soit comme le rapport pondéré des quantités produites sur le volume des facteurs de production utilisé, soit comme le rapport pondéré du coût des facteurs sur le prix des produits. Remarquons que le recours à une organisation et à une technique plus efficientes n'augmente pas seulement le produit physique pour un niveau donné d'intrants : elles diminuent également le prix des productions relativement au coût des facteurs et, ensuite, créent des profits et des rentes plus élevés, après correction des variations des prix et des salaires avec les indices $\mathbf{P}$ et $\mathbf{C}$. Si un exploitant habile réussit à augmenter sa productivité, il augmentra son profit qui, éventuellement, augmentera à terme la rente payée au propriétaire. Si d'autres l'imitent, le prix du blé peut tomber mais la PTF, qui est le rapport pondéré du coût des facteurs sur le prix des produits, augmentera encore. Par ailleurs, un simple changement dans les salaires, les rentes et les prix en réponse à une tension démographique ou à une inflation n'affectera pas la PTF. Si par exemple la population augmente, les rentes peuvent s'élever relativement au niveau des prix agricoles pendant que les salaires diminuent. Là encore, l'indice de la PTF, s'il est correctement calculé, restera le même (8).

\section{CE QUE SUPPOSE LE CALCUL DE LA PRODUCTIVITÉ TOTALE DES FACTEURS}

La méthode pour estimer la PTF est évidemment susceptible de multiples critiques. Certaines sont techniques et sont discutées par ailleurs. D'autres portent sur le bien-fondé de l'usage des baux en histoire moderne : comment se combinent les redevances en nature et les rentes en argent ? Comment intégrer les redevances seigneuriales et le paiement des dîmes? Les rentes exprimées dans les baux sous-estimentelles la vraie rente foncière pour alléger la charge fiscale ? Heureusement, aucun de ces problèmes n'apparaît insurmontable avec notre échantillon (9).

Les hypothèses sous-jacentes à l'ensemble de l'exercice sont beaucoup plus importantes en ce qu'elles peuvent susciter beaucoup d'hostilité de la part des historiens. On suppose en l'occurrence que la technologie agricole est connue; le marché des produits existe; le marché de la location des terres est compétitif. Ces hypothèses exigent un examen particulier, d'autant plus qu'elles seraient difficilement justifiables pour toutes les régions de France. 
La première hypothèse est que nous sommes capables de reconstituer les principales caractéristiques de la technologie agricole. En d'autres termes, il nous faut savoir quelle quantité de travail une exploitation moyenne du Bassin Parisien utilise, quel volume de capital (semences, bétail, outils) est investi, et quelle est sá production annuelle. Ces informations sont nécessaires pour calculer la part de chaque facteur de production et de chaque production qui rentrent dans l'équation de la PTF. Elles peuvent être trouvées dans les traités d'agriculture de l'Ancien Régime et dans les archives des exploitations; il faut, certes, se méfier des variations entre fermes mais elles ne semblent pas d'une ampleur telle qu'elles puissent modifier en profondeur les calculs de la PTF (10).

La définition de la PTF suppose également l'existence de marchés rudimentaires sur lesquels les facteurs de production sont achetés et les produits vendus. Nous devons être capables de mesurer les prix de marché, préalable nécessaire au calcul des indices $\mathbf{C}$ et $\mathbf{P}$. Il n'est pas cependant nécessaire que toutes les activités de l'exploitant passent par le marché : il est sans importance, par exemple, que des membres de la famille travaillent à la ferme pourvu que des valets et des domestiques soient aussi engagés ; peu importe, de même, qu'une partie de la récolte soit autoconsommée si le reste est vendu. La chose importante est que l'exploitation soit pour une part impliquée dans les activités du marché car alors les coûts et les prix auxquels l'exploitant fait face sont ceux du marché, même pour la part qui reste extérieure à ce dernier. Seule cette équivalence est nécessaire, non une pleine et totale dépendance par rapport au marché. Cette proposition, évidemment, peut contrarier l'historien car les exploitations de l'Ancien Régime sont souvent considérées comme auto-suffisantes, c'est-à-dire à peu près isolées des circuits de distribution. Les sources, cependant, suggèrent que cette autosuffisance est largement un mythe, en particulier pour le Bassin Parisien. Hormis dans les rares paroisses possédant d'importants terrains communaux ou un sol particulièrement fertile, l'auto-suffisance requiert une terre d'au moins dix hectares et presqu'aucun paysan ne possède une telle surface autour de Paris. Il existe certes des vignerons indépendants mais ils produisent du vin pour le vendre au marché. Presque tous les autres, dans le Bassin Parisien, travaillent en plus sur de grandes exploitations ou louent des parcelles sur un marché de la terre assez tendu. En aucune manière, il sont auto-suffisants (11).

Pour survivre, donc, beaucoup de paysans sont contraints de vendre leur propre travail. Ils consacrent bien sûr beaucoup de temps à leurs propres champs mais, désireux d'obtenir en plus un salaire, ils sont aussi partiellement engagés sur le marché du travail. Une telle assertion choquera ceux qui conçoivent le salaire comme une invention du capitalisme moderne mais le fait est que des marchés de ce type existent dès le $X^{\mathrm{e}}$ siècle, si ce n'est plus tôt. Si l'on examine les rôles fiscaux de la fin du $\mathrm{XVI}^{\mathrm{e}}$ siècle ou du début du XVII ${ }^{\mathrm{e}}$ siècle, par exemple, on découvre une armée de travailleurs agricoles qui ne possèdent ni ne louent assez de terres pour survenir à leurs besoins. Journaux et livres de compte nous permettent de les entrevoir quand ils travaillent dans une ferme; les rotations fréquentes et le fait que beaucoup viennent de loin 
sont peu compréhensibles sans l'intervention d'un marché du travail. Au $\mathrm{XVI}^{\mathfrak{c}}$ siècle, beaucoup des travailleurs non qualifiés de Paris participent à la récolte dans les campagnes environnantes et nombre d'artisans du bâtiment parcourent de grandes distances à la recherche d'emplois. Au $\mathrm{XVIII}^{\mathrm{c}}$ siècle, et probablement bien avant, des saisonniers quittent des régions aussi lointaines que le Limousin pour couper les blés du Bassin Parisien (12).

Beaucoup de migrants parcourent de plus courtes distances, allant d'un village à l'autre au sein de leur pays, mais ces brefs circuits suffisent à créer un rudimentaire marché du travail. On peut cependant douter de la possibilité de mesurer des salaires, tant ces derniers semblent varier d'une manière totalement incompatible avec l'hypothèse de leur formation sur un marché parfait, créateur d'un taux unique. A Paris au $\mathrm{XVI}^{\mathrm{e}}$ siècle, par exemple, un travailleur du bâtiment non qualifié peut gagner 7 sols par jour quand un autre qui travaille à ses côtés obtient presque trois fois plus. Dans une ferme, un valet nouvellement engagé augmentera son salaire d'un tiers s'il reste une seconde année. De telles variations reflètent sans doute des différences de qualification ou de force; elles sanctionnent une relation de confiance, prisées par les maîtres de cette époque qui répugnent à confier leurs récoltes et leurs troupeaux à des serviteurs irresponsables. Un plus haut salaire pour un charretier qui sert depuis plus d'un an ou pour une fille d'étable dont la famille est connue du fermier est ainsi tout à fait compréhensible. Le fermier se fie aux services rendus et à la réputation pour sélectionner ses domestiques. Son comportement diffère peu de celui d'un employeur d'aujourd'hui qui s'appuie sur des références et sur l'expérience du candidat. Ce que nous estimons être des variations étonnantes dans les salaires n'est donc que le résultat du fonctionnement normal du marché du travail dont l'efficacité est suffisante pour établir de fines distinctions entre employés (13).

Il reste le problème de la mesure effective des salaires. L'idéal serait d'avoir celui d'un travailleur de ferme, si possible non qualifié. II est cependant difficile à apprécier car d'une part les domestiques reçoivent souvent une part importante de leur rémunération en nature, et d'autre part, celle-ci varie beaucoup selon la saison et la tâche à accomplir. La seule alternative est d'utiliser des salaires urbains de travailleurs du bâtiment non qualifiés. Leur valeur modale nous indique ce que gagne un journalier du bâtiment non qualifié et permet de dépasser les différences de force ou de qualification. On peut objecter que les salaires urbains diffèrent de ceux de la campagne mais les preuves du $\mathrm{XV}^{\mathrm{c}}$ et $\mathrm{XVI}^{\mathrm{c}}$ siècles pour la région autour de Paris suggèrent que la différence, pour les journaliers non qualifiés, est minime, au moins durant la récolte quand les fermiers embauchent de la main d'œuvre temporaire. "Au $\mathrm{XVI}^{\mathrm{e}}$ siècle, le salaire de deux manouvriers, dont l'un travaillait aux champs et l'autre dans la ville, était identique ", écrit M. Baulant, et les données qu'elle a rassemblées appuient son assertion. Un aide urbain non qualifié gagne 2,5 sols par jour en 1500-1505 et 10,4 en 1594-1598; un hotteur pendant la récolte de raisin reçoit 2,5 sols par jour en $1500-1505$ et 10 en $1594-1595$ (14). 


\section{Histoire \& Mesure}

Même s'il existe parfois des différences entre les salaires urbains et ruraux, la tendance est presque partout la même pour les travailleurs sans qualification; or c'est cette tendance, et non des prix absolus, qui importe pour déterminer les changements dans l'indice $\mathrm{C}$ et, de là, dans la formule de la PTF. Pour les domestiques ruraux et les journaliers, pour les aides citadins du bâtiment et pour beaucoup d'autres emplois non qualifiés, les salaires évoluent en parallèle, si l'on se fie du moins aux séries disponibles pour la région parisienne. C'est vrai pour l'aide de la ville et le hotteur rural entre 1500 et 1598 ; selon M. Baulant, c'est vrai durant cette période pour presque tous les travaux (15).

Il est évidemment exclu d'en conclure qu'il existe un marché national de l'emploi. Ce type de marché reste régional, même si celui autour de Paris est assez vaste pour inclure les localités desquelles nos baux dépendent. De plus, il ne faut pas sous-estimer la segmentation de ces marchés du travail, avec, par exemple, des fermiers capables parfois de traiter des embauches pour un salaire très inférieur à celui pratiqué à Paris. Peut-être également que les rémunérations rurales au XVIII ${ }^{\mathrm{e}}$ siècle ne suivent plus celles des villes d'aussi près que deux siècles auparavant.

Cependant, s'il existe des preuves d'une certaine fragmentation en l'état actuel de la recherche, elles ne sont ni déterminantes ni convaincantes. D'abord, une telle fragmentation est sans doute plus un phénomène du XIX ${ }^{\mathrm{e}}$ siècle que de l'Ancien Régime. Ensuite, les différences de rémunération d'une place à l'autre ne sont pas considérables et elles peuvent simplement refléter l'hétérogénéité des emplois, la complexité des paiements en nature et les variations du coût de la vie. Enfin, il est difficile de prétendre qu'il existe une complète segmentation quand l'on constate cette énorme mobilité du travail dans le Bassin Parisien sous l'Ancien Régime : la main d'œuvre de la capitale aide aux récoltes, les domestiques quittent les fermes pour la ville de même que nombre de pauvres qui fuient la misère de la campagne. Beaucoup de familles entières, de même, viennent et repartent des petites agglomérations autour de Paris, sans doute à la recherche de travail. Une telle mobilité exclut que le marché régional du travail soit divisé en compartiments isolés et étanches (16).

La méthode utilisée requiert également l'existence de marchés et de coûts chiffrés pour le capital agricole, en particulier le bétail. Heureusement, les marchés à longue distance pour les chevaux et les moutons, par exemple, remontent beaucoup plus loin dans le passé. S'il est vrai que les séries de prix pour les animaux sont médiocres et que, de plus, les différences entre races obligent à la prudence, il est malgré tout possible de rassembler les séries nécessaires pour estimer l'évolution du coût de ce facteur, au moins pour des moyennes sur des périodes de 25 ans qui suffisent pour établir l'indice $C$ de la formule du PTF (17).

Pour l'indice des prix $\mathbf{P}$, il faut connaître le prix des productions agricoles pour lequel les grains soulèvent les difficultés les plus intimidantes. Les grains se vendent et s'achètent depuis longtemps mais les problèmes de transport réduisent beaucoup l'étendue du marché. De plus, le coût élevé du stockage et de la préparation pour la vente ont 
pour effet de limiter l'offre de court-terme, ce qui, combiné à une demande inélastique, a pour effet de rendre les prix volatiles et difficiles à mesurer. Parallèlement, il semble que beaucoup de grains ont échappé au marché, passant au consommateur par le biais de paiement en nature ou tout simplement par l'auto-consommation. On peut donc supposer que le prix du grain à la ferme n'a pas de relation avec le prix du marché qui rentre dans l'indice $P$ (18).

Là encore, la difficulté n'est pas insurmontable. Il serait, en effet, absurde de prétendre que les paiements en nature créent l'équivalent d'un second marché sur lequel le prix des grains serait sans relation avec celui du marché ouvert. Les chanoines de Notre-Dame reçoivent de nombreuses redevances en nature mais ils les évaluent avec le prix courant du marché (de Paris si les grains y sont apportés ou des marchés locaux dans le cas contraire). Et quand vient le moment de vendre les stocks, les chanoines observent l'état du marché pour apprécier ce que leurs grains rapporteront (19). En conséquence, les paiements en nature ne semblent pas constituer un marché autonome et séparé, d'autant plus que les droits perçus directement en nature (comme certaines dîmes, par exemple) sont souvent achetés contre argent. Le même raisonnement permet de rejeter l'hypothèse que le prix des grains à la ferme n'est pas lié à celui du marché.

Les dernières difficultés posées par le prix des grains peuvent également être résolues. Le calcul de moyennes de longue durée diminue leur volatilité et, s'il est vrai que les coûts de transport différencient les prix sur les marchés à longue distance, on peut supposer que ces moyennes (qui suffisent pour l'indice $P$ ) tendent à évoluer de manière identique, au moins tant que ces lieux de vente ne sont pas trop éloignés les uns des autres. Autour de Paris, les prix sur les marchés locaux aux $\mathrm{XVI}^{\mathrm{c}}$ et $\mathrm{XVII}^{\mathrm{e}}$ siècles sont plus bas et moins stables qu'à Paris mais leur tendance est similaire dans un rayon de cent kilomètres, en particulier si l'on compare les moyennes qui lissent les séries et les rendent comparables. Ces mouvements parallèles ne sont guère surprenants car nombre d'indices montrent que les marchands et même les gros fermiers font circuler des quantités suffisantes pour opérer un arbitrage entre-marchés devant les trois siècles de l'Ancien Régime. Avec de tels agents économiques qui achètent ou vendent selon l'écart des prix, il est peu étonnant que la tendance de ces derniers soit identique malgré des niveaux absolus différents (20).

L'ultime hypothèse nécessaire est que les profits non taxés $\mathrm{du}$ fermier retournent au propriétaire, en d'autres termes que le marché de la rente foncière est compétitif, sans barrière à l'entrée. Ce n'est certainement pas vrai dans le court terme car la renégociation des baux exige du temps de même que, plus simplement, la prise de conscience par le propriétaire qu'il peut obtenir plus de ses terres. Mais seul le long terme nous concerne ici. Il est également possible de suppposer que les tenanciers puissants maintiennent les rentes à un bas niveau et retient ainsi un plus grand profit même en longue durée. On pense aux fermiers décrits par G. Lefebvre dans les Paysans du Nord qui, grâce aux coutumes du " mauvais gré " ou du "droit de marché " empêchent les 
propriétaires de les évincer ou d'augmenter les rentes. Or le Nord n'est pas la seule région où les fermiers détiennent un tel pouvoir; dans le Bassin Parisien, par exemple, les exploitants réorganisent subrepticement les terres et des dynasties de riches fermiers monopolisent l'attribution des grandes fermes (21). A la différence des marchés du travail, des animaux de ferme ou des produits agricoles qui essaiment avec des centaines de petits acteurs, le marché de la location des terres dans chaque village ne concerne qu'un petit nombre de fermiers puissants et n'est pas animé par une vive concurrence.

Cependant, le pouvoir de tels fermiers a probablement été exagéré. G. Lefebvre lui-même l'a souligné avec prudence; le « mauvais gré » n'est pas pratiqué dans tout le Nord. Quand il existe, il ne s'applique qu'à des droits de propriété particuliers, eux-mêmes souvent inconnus dans une large partie du Bassin Parisien, là où sont situées les propriétés de Notre-Dame de Paris (22). De plus, les chanoines, comme certains autres propriétaires, tolèrent les réarrangements de parcelles entre tenanciers dans la mesure où les terrains restent assez bien identifiés pour ne pas obscurcir la répartition des droits. Et d'ailleurs pourquoi refuseraient-ils de tels échanges, dans la mesure où ils conservent un signe tangible de leur droit de propriété, si l'efficience des exploitations s'en trouve augmentée (23) ? Il est de même probable que si les riches tenanciers pesaient ainsi sur les rentes, on trouverait de fréquentes mentions de cette question dans les manuels de propriétaires et dans les textes d'agronomie. Or, on les chercherait en vain alors même que les auteurs consacrent une attention considérable à la relation avec les fermiers. Charles Estienne, par exemple, qui écrit au milieu du XVI ${ }^{e-}$ siècle, parle des tricheries de ces derniers, des dommages qu'ils peuvent créer s'ils ne sont pas assez surveillés, des qualités nécessaires qu'il faut exiger d'eux et comment ils doivent être récompensés, mais jamais il ne suggère qu'ils excercent quelque pouvoir sur la fixation de la rente ni que leur éviction pose problème. Le même refrain est repris par les auteurs des deux siècles suivants: loin de se plaindre de l'impossibilité de se séparer d'un fermier puissant, ils insistent sur l'intérêt financier qu'il y a à récompenser et à retenir les meilleurs. L'existence d'une dynastie de ces derniers peut donc signifier moins leur puissance que leur talent, talent qui en fin de compte profite au propriétaire (24).

Une constatation qui a souvent été interprétée comme le signe d'un pouvoir de marché exercé par les fermiers est que la rente par hectare est plus faible pour les grandes exploitations que pour les parcelles (25). Cet argument n'est convaincant que s'il prend en compte la qualité des sols, leur localisation et les autres facteurs qui influencent la rente. La vérification est envisageable avec notre échantillon en recourant à une analyse de régression. Elle permet de calculer dans quelle proportion une variable donnée dépend d'autres variables : dans le cas présent, nous voulons estimer le degré de la dépendance du logarithme de la rente par hectare par rapport à la taille de la propriété, à la qualité du sol, à la localisation et à d'autres facteurs de ce type. L'information requise peut être glanée dans les baux et dans les descriptifs d'exploitation. La régression montre que les plus importantes fermes de Notre-Dame se louent effectivement pour un prix moindre à l'hectare. Un doublement 
de la superficie réduit la rente de $9 \%$, toutes les autres variables égales par ailleurs (Tableau 1, régression 1) (26).

Il serait pourtant faux d'en conclure que les fermiers de NotreDame font pression sur la rente car une autre explication semble beaucoup plus convaincante qui ne fait pas référence à la notion de pouvoir de marché. Il faut rappeler que la location d'une terre, même pour une rente fixe, implique des risques pour le propriétaire: sa propriété peut être ruinée par négligence, le fermier peut partir sans payer son bail... De tels risques sont loin d'être insignifiants, même pour de petites parcelles (27). Le propriétaire est parfois contraint d'intenter un procès ou de saisir les biens du tenancier. Or seuls les riches exploitants possèdent des biens, en troupeaux ou en équipement, suffisants pour garantir une dette. Avec les petits paysans, au contraire, ces assurances n'existent pas et le seul recours est d'exiger une prime de risque sous la forme d'une rente plus élevée. Cette dernière est donc la compensation des risques encourus avec des tenanciers sans garantie financière.

D'autres éléments ajoutent encore au doute sur le pouvoir de marché des fermiers du Bassin Parisien. Il est fréquent que les gros exploitants de la région changent de fermes au cours de leur carrière. Cette mobilité signifie que les propriétaires introduisent facilement des concurrents venus d'autres villages. Les fermiers ont également de grandes familles et si l'on peut imaginer une collusion entre deux ou trois patriarches en vue de déprimer localement les rentes, une telle collusion s'effondrerait sûrement quand ces chefs de familles auraient à installer dans des fermes leurs nombreux enfants (28). Finalement, si les dynasties de fermiers contrôlent le mouvement de la rente et s'approprient une partie des profits non taxés, alors l'augmentation du fermage doit être significativement plus faible quand un même exploitant (ou son parent) renouvelle le bail, et plus forte quant un concurrent prend le relais. Or sur notre échantillon, une telle constatation n'apparaît pas. La régression du taux de croissance entre chaque bail du logarithme de la rente sur le taux de variation du rapport entre les prix agricoles et le coût des intrants et sur les autres variables qui affectent le fermage montre que le maintien du même exploitant a pour effet de diminuer la rente d'un montant très faible et statistiquement insignifiant (Tableau 2, régression 1). Si un parent de l'ancien détenteur du bail le renouvelle, l'effet est tout aussi limité.

Un tel résultat ne doit pas surprendre. Les propriétaires revoient les baux tous les neuf ans, avec une préoccupation d'abord tournée vers le profit. Mises à part les régions de "mauvais gré ", rien ne l'empêche de se tourner vers un nouveau fermier. L'absence de barrières à l'entrée exclut ainsi que les tenanciers ne retiennent une part excessive de profit. Il est évidemment permis de s'interroger sur la notion de profit entrepreneurial. Notre hypothèse est que, dans le long terme, la compétition du marché le réduira à zéro, ne laissant au fermier que l'équivalent de ce qu'il avait pu gagner sur le marché du travail et égalisant ainsi le coût de l'activité de l'entrepreneur à un taux de salaire. Bien qu'une telle supposition soit susceptible de critique, l'évidente 
compétition sur le marché de la rente foncière plaide en sa faveur. L'analyse des gains d'une ferme au cour du XVIII ${ }^{\mathbf{c}}$ siècle suggère de même que les profits de l'entrepreneur sont très minimes et en moyenne beaucoup trop faibles pour affecter le calcul de la PTF (29).

Bien que l'utilisation des prix et des baux semble désormais raisonnable, le lecteur attend peut-être une preuve plus tangible de ce que la PTF produit des résultats fiables. Sur un cas particulier, grâce à un ensemble peu commun de données, il a été possible de procéder à une mesure physique des quantités produites et des facteurs utilisés pour une ferme du Bassin Parisien au XVIII ${ }^{\mathbf{c}}$ siècle : or le calcul de la PTF sur les mêmes données aboutit à des résultats très similaires. L'emploi des prix à la place des quantités physiques paraît donc acceptable (30).

\section{LES PROPRIÉTÉS DE NOTRE-DAME}

\section{a) L'évolution de la productivité}

Quels enseignements nous livrent les baux? Examinons d'abord l'évolution de la PTF. Rappelons qu'elle est à égale à $(\mathbf{r}+\mathbf{t})^{\mathrm{s}} \mathbf{C} / \mathbf{P}$, avec : $\mathbf{r}$, la rente par hectare ; $\mathbf{t}$, les charges fiscales par hectare ; $\mathbf{s}$, la part de la terre dans les facteurs de production ; $\mathbf{C}$, un indice géométrique pondéré du coût des autres facteurs de production; $\mathbf{P}$, un indice géométrique du prix des produits agricoles pondéré selon leur part dans le revenu total. Si nous laissons de côté les impôts pour l'instant - une simplification corrigée plus tard -, alors le logarithme de la PTF sera presque égal à In $\left(\mathbf{r}^{\mathbf{s}} \mathbf{C} / \mathbf{P}\right)$, dont on peut calculer la moyenne pour l'ensemble des propriétés. La même opération pour des périodes différentes retrace, au moins approximativement, l'évolution de la PTF.

La figure 2 dessine cette évolution par périodes de 25 ans. Une correction mineure pour tenir compte des variations de la qualité de la terre en fonction de la composition changeante de l'échantillon est incluse dans le graphique (31). Ce dernier présente également les résultats d'un ajustement opéré pour intégrer les impôts omis dans le calcul de la PTF. Cet ajustement est d'autant plus nécessaire que la monarchie impose une charge fiscale de plus en plus lourde sur la terre. La sur-estimation initiale est cependant très limitée (32).

Les années 1450-1519 sont exclues car les séries de prix sont d'une trop médiocre qualité et le nombre de baux utilisables diminue. Il est bien connu que cette période connaît une récupération après les dévastations de la guerre de Cent Ans. Des tenanciers réoccupent les fermes abandonnées, reconstruisent les murs, les écuries, débroussaillent les champs. Ce processus se poursuit très avant pendant le XVI siècle, en particulier dans les villages dotés de sols médiocres ou situés loin de Paris. En 1545, par exemple, Notre-Dame défriche encore des terres dans le village de La-Grande-Paroisse ( 77 kilomètres au sud-est de 


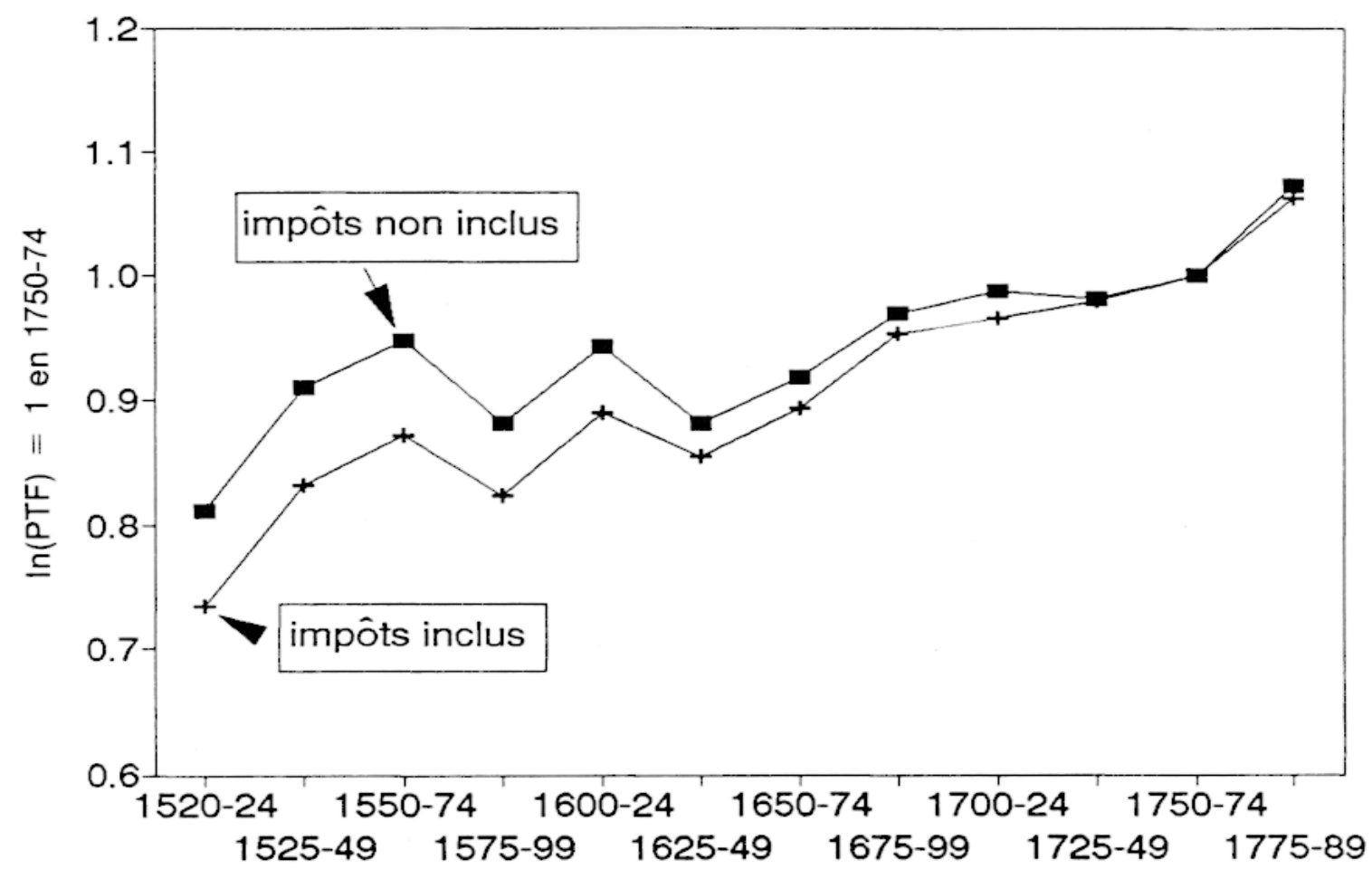

Figure 2: Evolution de In (PTF).

Paris) où l'un de ses fermiers, Jean Godet, doit éclaircir un champ de bruyères de 9 hectares. Godet doit également enclore le pré avec des fossés pour le protéger des animaux errants, preuve que le processus s'est poursuivi au-delà d'une simple récupération et devient une opération plus vaste d'amélioration des terres (33). La persistance de ce mouvement durant tout le siècle pourrait expliquer les hauts niveaux relatifs de l'indice de la PTF en 1550-74 (fig. 2). Les investissements dissimulés derrière ces améliorations auraient stimulé la rente foncière et apparaîtraient, un peu par erreur, sous la forme d'une PTF plus élevée. Ce sont eux qui expliqueraient également le taux de croissance élevé. Entre 1520-1524 et 1570-1574, l'indice augmente de plus de 0,3\% par an, résultat impressionnant qui se compare avantageusement aux performences anglaises du XVIII ${ }^{c}$ siècle.

Selon toute vraisemblance, cependant, la raison de cette forte productivité en 1570-1574 ne réside pas dans la récupération d'après la guerre de Cent Ans. Les informations contenues dans les baux permettent souvent de déduire la fraction de la rente qui résulte d'améliorations, au moins quand des bâtiments sont concernés et, cette déduction faite, les rentes et donc la PTF changent à peine. On dira que les investissements consacrés aux améliorations foncières ne figurent pas dans les baux. Mais il est peu probable que les défrichements par exemple aient été poursuivis au-delà de 1550 , en particulier dans les propriétés proches de Paris, où le niveau de la PTF en 1550-1574 est le plus élevé. Les fermes autour de Paris ont beaucoup moins souffert 
pendant la guerre de Cent Ans; leur remise en état est donc surtout l'œuvre du XV $\mathrm{XV}^{\mathrm{e}}$ siècle, poursuivie peut-être jusqu'en 1525 mais certainement pas jusqu'en 1550 (34). D'autres forces poussent donc la PTF vers le haut, des forces qui se font plus intenses près de la capitale. Peut-être sont-elles dues aux avantages offerts par une grande ville, un aspect sur lequel nous reviendrons.

Après les sommets du milieu du siècle, la PTF s'effondre avec les guerres de Religion. Entre 1550-1574 et 1575-1599, elle perd presque $5 \%$, quantité considérable dans l'Europe ancienne où les croissances de la productivité agricole les plus remarquables ne dépassent pas quelques dixièmes de pourcent par an. La guerre est sans conteste la cause de cet effondrement. Le déclin est plus marqué pendant et juste après les années 1589-1594, période des combats les plus intenses dans le Bassin Parisien, quand des troupes indisciplinées traversent la région et sèment la devastation. Non seulement ils piétinent les récoltes, s'emparent du bétail et les grains, brûlent les bâtiments, mais en plus ils pratiquent des extorsions, le rapt et interrompent le commerce. Il est compréhensible que beaucoup de fermiers choisissent la fuite, abandonnant leur exploitation au pillage. Bernaye, tenancier de Notre-Dame, laisse son bail de La-Grande-Paroisse en 1594 à cause des attaques de bandes de soldats, et la guerre laisse la ferme de Dampmart abandonnée et ruinée en 1597. Pendant cette période d'anarchie, la PTF perd peut-être $25 \%$ (35).

Telles sont les lourdes conséquences de la guerre. L'indice calculé peut cependant sembler mal adapté pour juger des effets d'événements si transitoires puisqu'il a été établi pour mesurer la seule tendance de longue durée. Pourtant, les faits suggèrent que son écroulement au cours des guerres de Religion est bien réel. Ce qui fait chuter l'indice dans les années 1590, en effet, ce n'est pas le déclin de la rente mais un brutal retournement des prix agricoles. Cet effondrement est lui-même le signe d'un marché perturbé et de productions anéanties (36).

La productivité reprend fortement dans les années de paix qui ouvrent le $\mathrm{XVII}^{\mathrm{e}}$ siècle. Ensuite, elle décline dans le second quart du siècle. Cette diminution de la PTF résulte probablement de la lourde fiscalité foncière imposée par l'engagement royal dans la guerre de Trente Ans. Or, l'ajustement de nos calculs ne porte que sur la portion des profits ou des surplus du fermier qui vont au fisc plutôt qu'au propriétaire. Ces impôts qui montent en flèche ont sans doute aussi exercé des ravages en cassant l'économie rurale: l'endettement des paysans conduit souvent à la saisie du bétail et du capital agricole. En plus des mouvements de troupe pendant la Fronde et d'une série de désastreuses récoltes au début des années 1630, les crises liées au prélèvement fiscal dessinent la chronologie de la baisse de la PTF en 1625-1649 (37).

Le siècle qui suit connaît une restauration, puis une croissance lente. Une partie au moins des gains apparents après 1650 est en fait artificielle car ils reflètent une baisse du coût des transports et non une augmentation de la productivité agricole. Rappelons que le coût du transport crée un écart conséquent entre le prix à la ferme et celui à Paris pour les marchandises pondéreuses comme le grains, et donc réduit d'autant plus 
la rente que l'on s'éloigne de la capitale. Comme nos calculs de la PTF s'appuient sur des prix parisiens et sur des rentes locales plus faibles, ils surestiment le niveau de la productivité pour des fermes situées à la périphérie puisque la rente foncière est fixée en tenant compte des prix locaux.

Tant que les prix locaux des grains évoluent en parallèle à ceux de Paris - c'est le cas normal _- il n'y a pas d'inquiétude à avoir quant à la fiabilité de l'estimation. Bien que les niveaux absolus de la PTF marquent parfois de légères oscillations erratiques, les tendances de la productivité et de son taux de croissance sont les mêmes. Mais à la fín du XVII ${ }^{\mathrm{e}}$ siècle et au début du XVIII ${ }^{c}$ siècle, les prix sur les marchés locaux comme ceux de Pontoise et de Soissons augmentent progressivement pour approcher le niveau parisien et le différentiel se réduit (38). La cause en est la baisse des coûts de transport comme le prouve le fait que l'augmentation des prix locaux relativement à ceux de Paris est plus accentuée à mesure que l'on s'éloigne de la capitale. Cette baisse est elle-même la marque d'une meilleure productivité, mais dans les transports plus que dans l'agriculture (39). Malheureusement, la mesure de la PTF confond les deux. Les rentes augmentent quand les prix locaux convergent vers ceux de Paris mais, comme elles sont rapportées à l'indice $\mathbf{P}$ obtenu à partir des prix parisiens, il s'en suit une hause de la PTF, particulièrement sensible sur les fermes périphériques. Or, ce sont précisément ces dernières qui semblent gagner le plus en productivité à la fin du XVII ${ }^{\mathrm{e}}$ siècle.

L'observation des prix sur les marchés à l'extérieur de Paris peut indiquer quelle est la part de l'augmentation de la PTF entre 1650-1674 et 1750-1774 qui relève de la baisse des coûts de transport et de la hausse concomitante des prix locaux. Considérons, par exemple, un marché éloigné, sur lequel le changement dans l'évolution des prix par rapport à ceux de la capitale est important. Soissons est un parfait exemple : situé à près de 100 kilomètres de Paris, il est plus distant de la capitale qu'aucune des propriétés de notre échantillon. C'est sans surprise que l'on constate que le différentiel des prix explique $8,3 \%$ de la hausse de la PTF entre 1650-1674 et 1750-1774, soit les trois quarts du gain total de $11,3 \%$. Plus près de Paris, l'effet de la convergence des prix est moins sensible. A Pontoise, distant de 30 kilomètres, elle n'explique que $3,6 \%$ de la même mesure de la PTF. Or, ce dernier cas est plus représentatif par sa proximité de Paris de l'ensemble de l'échantillon. En conséquence, la croissance réelle de la PTF entre 1650-1674 et 1750-1774 est seulement d'environ $7,7 \%$. Les $3,6 \%$ qui relèvent de l'amélioration des transports ne sont pas cependant à négliger car ils contribuent autant que les progrès agricoles à nourrir la population parisienne en extension.

Après un siècle de croissance faible, la PTF devient plus dynamique à la fin du XVIII ${ }^{c}$ siècle. Entre $1750-1774$ et $1775-1789$, elle gagne 6,5\% si l'on prend la moyenne des propriétés. La pointe de la fin de l'Ancien Régime est encore plus perceptible si l'on regarde les taux de croissance. Ils dépassent $0,3 \%$ par an et atteignent un pic de plus de $1 \%$, taux comparables ou supérieurs à ceux de l'Angleterre. De fait, au début du $\mathrm{XVIII}^{\mathrm{c}}$ siècle, quand la croissance de la PTF anglaise semble monter en 
flèche, elle gagne $0,6 \%$ par an selon N.F.R. Crafts; plus avant dans le siècle, ses estimations la font tomber à environ $0,2 \%$. Le travail de $R$. Allen sur les Midlands s'accorde à peu près avec un chiffre entre $0,2 \%$ et $0,3 \%$ pour l'ensemble des $X V I^{c}$ et $X V I I I^{c}$ siècles (40). L'agriculture du Bassin Parisien n'a ainsi qu'un très faible retard sur l'Angleterre.

Cette performance n'est-elle pas cependant que le fruit d'une illusion? Ne reflète-t-elle pas, au moins en partie, une diminution des coûts de transport comme avant 1750 ? La réponse est non. D'abord, l'indice de la PTF après 1775 ne croît pas plus vite sur les propriétés éloignées que sur celles près de Paris, à la différence de la période précédente. Ensuite, les prix locaux cessent après 1750 d'augmenter plus vite que ceux de la capitale : leur mouvement ne peut plus rendre compte d'aucune augmentation de la PTF. Les prix à Soissons expliquent peut-être 1,8\% de la hausse de la PTF entre 1750-1774 et 1775-1789; ceux de Pontoise - les plus significatifs pour l'échantillon - absolument rien.

La mesure utilisée, il est vrai, peut marquer un léger décalage par rapport à la réalité. Il faut du temps pour renouveler un bail ou pour percevoir la prospérité d'un tenancier qui pourrait supporter une rente plus lourde. Un propriétaire avisé peut préférer attendre avant d'exiger une augmentation de peur que son fermier fasse faillite. Les chanoines de Notre-Dame, par exemple, enquêtent sur plusieurs tenanciers à la fin des années 1750 et découvrent qu'ils sont très prospères ce qui les incite à écarter la crainte d'une faillite. C'est seulement alors que commence la montée de la rente. Si un tel cas de figure apparaissait général, cette dernière pourrait être plus précoce que ne le suggèrent les graphiques (41).

Cependant, que le retournement se produise un peu plus tôt ou un peu plus tard ne change rien au fait que la hausse de la PTF au $\mathrm{XVIII}^{\mathrm{e}}$ siècle n'est pas réservée aux propriétés de Notre-Dame. Des croissances nominales de la rente de $79 \%$ à $120 \%$ entre 1730 et 1780 sont fréquentes en Ile-de-France, Picardie ou Beauce. Or le chiffre est de $105 \%$ pour notre échantillon. Puisque la tendance des prix et de la fiscalité est la même aux environs de Paris, les estimations de la PTF peuvent avoir une portée plus générale pour la région (42).

\section{b) Les résultats des régressions.}

L'analyse de régressions appuyées sur les baux individuels corrobore cette évolution sur trois siècles de la productivité et nous aide à préciser les résultats. Les variables dont on cherche à expliquer l'allure sont le logarithme de la PTF et son taux de croissance, les deux calculés bail par bail. Il est possible de corriger les effets liés à la qualité de la terre et à sa localisation sur la rente et, de là, sur notre indice de la PTF en incluant ces deux facteurs parmi les variables explicatives des régressions. En retour, ces dernières aideront à déterminer si les 
changements de la PTF ou de son taux de croissance sont seulement des hasards statistiques (dûs par exemple à la maigreur de notre échantillon) ou s'ils expriment une réalité.

La régression avec In (PTF) confirme le plongeon de la PTF au cours de la période la plus dure des guerres de Religion. Le coefficient de la variable indicatrice pour les terribles années 1589-1597 (période des combats les plus intenses autour de Paris, avec ses conséquences sur le cycle de 3 ans des récoltes) se traduit par une chute de $25 \%$ de la PTF et la statistique est trop élevée pour qu'il s'agisse d'un accident (Tableau 1, régression 2). De la même façon, la PTF bondit littéralement après 1775 , de $6,6 \%$ si l'on en juge d'après le coefficient de la variable pour les années après 1775. Comme pour les 3 autres siècles, le coefficient de l'année, qui prend la moyenne de la croissance de la PTF en dehors des périodes $1589-1597$ et $1775-1789$ est consistante avec notre analyse présentant des gains rapides au début du XVI ${ }^{\mathrm{c}}$ siècle, une vive récupération après 1589-1597, une crise en 1625-1650 et une croissance lente ensuite (43).

De même que l'analyse des prix locaux aux $\mathrm{XVII}^{\mathrm{c}}$ et $\mathrm{XVIII}^{\mathrm{c}}$ siècles, les régressions autorisent à plaider contre une interprétation de l'indice de la PTF en termes exclusivement de baisse des coûts de transport. Si cette dernière seule devait être explicative, alors le taux de croissance de la PTF devrait apparaître plus élevée loin de Paris, là où les prix augmentent le plus. On devrait donc s'attendre à un coefficient positif pour le logarithme de la distance à Paris dans les régressions (Tableau 2 , régression 2). Or il n'en est rien : le coefficient est insignifiant, négatif. Au moment où les transports gagnent en efficience, l'économie agricole fait de même.

\section{COMMENT EXPLIQUER LA CROISSANCE DE LA PRODUCTIVITE?}

Qu'est-ce qui explique cette lente progression des années 1650-1750 ou les montées rapides observées au XVI ${ }^{\mathrm{e}}$ siècle, au début du XVII ${ }^{\mathrm{e}}$ siècle et après 1775 ? Une partie de la croissance de 1650 à 1750 reflète l'amélioration des transports et la poussée au début du XVI ${ }^{\mathrm{c}}$ siècle traduit l'effort de récupération après les guerres de religion. Mais pour le reste? La réponse n'est pas à chercher dans une révolution sociale ou technologique car rien de tel ne se produit avant 1789. Aucun mouvement d'enclosures ne dépeuple les campagnes et aucune innovation technique ne bouleverse les travaux agricoles, même à la fin du $\mathrm{XVIII}^{\mathrm{e}}$ siècle. Ce qui change, plutôt, provient de la possibilité de développer le commerce grâce à la proximité de Paris et de son marché en expansion. Les historiens ont souvent expliqué ce processus qui présente le marché urbain comme la cause des progrès agricoles avant que se manifeste le relais technologique à la fin du XIX ${ }^{\mathrm{e}}$ siècle.

Pour la région parisienne, qui possède un large marché en croissance, il est possible de comprendre comment l'agriculture bénéficie de la 
proximité citadine et des opportunités qu'elle offre pour la spécialisation. La plus forte productivité des fermes près de Paris ne provient pas nécessairement d'une remarquable hausse des rendements - les informations sur l'évolution de ces derniers manquent en fait de précision mais elle est en partie une réponse à la population animale croissante de Paris. Les chevaux attelés aux voitures des privilégiés, par exemple, tirent vers le haux les prix des fourrages et encouragent la production des aliments pour animaux près de la capitale. Assez vite, les fermiers développent les prairies artificielles pour nourrir leur propre bétail et ils portent en ville leur avoine, leur paille et leur foin. Ils peuvent en repartir avec des charges d'engrais à étendre sur leurs champs, désserant ainsi la terrible contrainte que fait peser le manque de fumures dans l'agriculture ancienne et stimulant les rendements. Tous ces changements sont de faible ampleur et accomplis à une petite échelle, sur une parcelle ou dans le coin d'un champ, plutôt que sur de vastes fermes. Ils n'en suffisent pas moins pour pousser la PFT vers le haut (44).

Les régressions soulignent le rôle important tenu par la proximité de Paris et par son développement. La multicollinéarité empêche d'ajouter la population parisienne dans les variables pour la régression $\mathrm{du}$ logarithme de la PTF mais la vitesse d'évolution de la population parisienne a un effet important sur le taux de croissance de la PTF (Tableau 2, régression 2). Au début du XVII ${ }^{\mathbf{e}}$ siècle, par exemple, quand la population gagne annuellement $1,3 \%$ - chiffre imposant pour les normes contemporaines-, cela induit $0,3 \%$ d'augmentation en plus pour la PTF (45).

La petite taille des exploitations est fréquemment invoquée depuis Arthur Young pour expliquer les échecs de l'agriculture française; il serait donc interéssant de savoir si ce facteur affecte également la PTF de la région parisienne. Les régressions semblent répondre par la négative. Des tailles élevées (mesurées par le logarithme de la surface de l'exploitation) diminuent effectivement la rente et ainsi la mesure de la productivité, mais l'effet traduit simplement - on l'a dit - le risque à couvrir par les tenanciers de petites fermes (Tableau 1, régression 1 et 2). Si l'on ajoute dans la régression une mesure quelque peu grossière du regroupement des terres (le logarithme du nombre d'hectares par parcelle de propriété), il semble qu'elle stimule le niveau de la PTF mais le coefficient pourrait n'être qu'un artefact dû à la multicollinéarité (Tableau 1, régression 3). Plus convaincantes peut-être sont les régressions sur les taux de croissance car la multicollinéarité est sans effet. Alors, ni la taille de la propriété, ni son remembrement ne semblent affecter la croissance de la PTF (Tableau 2, régression 2 et 3).

Encore faut-il ici être prudent. Ce qu'impliquent réellement les régressions sur les taux de croissance, c'est qu'aucun obstacle dans le long terme ne vient gêner l'élargissement ou le regroupement de propriétés. Rappelons que ce regroupement est fréquemment réalisé par des tenanciers qui louent des terres à différents propriétaires. Bien que les propriétés demeurent distinctes, les fermiers les travaillent comme s'il s'agissait d'un ensemble unique. Lorsque André-Paul Hanoteau en 1784 prend le bail d'une ferme de 30 hectares possédée par Notre-Dame à 
Tremblay-lès-Gonesse, elle ne constitue qu'une partie des centaines d'hectares qu'il cultive autour de Tremblay (46). De tels cumuls de baux se multiplient au XVIII ${ }^{\mathrm{c}}$ siècle et permettent des économies d'échelle. La pratique permet au tenancier de réduire les dépenses pour les bâtiments, l'équipement et pour certaines tâches (47), et d'exercer son talent d'entrepreneur sur de multiples propriétés.

Notre-Dame possède suffisamment de biens pour effectuer à l'occasion un regroupement en louant deux de ses terres au même fermier. Lorsque l'on examine de tels regroupements, on constate quelques échecs mais aussi quelques remarquables succès, comme à La Grande Paroisse au début du XVII ${ }^{c}$ siècle où la PTF gagne $6 \%$ (48). D'autres informations sont disponibles, tirées des rôles fiscaux, sur la taille des propriétés à la fin du XVIII ${ }^{\mathbf{c}}$ siècle. Les contributions fiscales sont revues chaque année pour rester proportionnelles à la superficie exploitée par un fermier et ainsi elles peuvent fournir une approximation du montant total de terre qu'il cultive. Si l'on compare de telles estimations pour plusieurs tenanciers à des moments différents, les changements donneront une mesure relative de l'accroissement de l'échelle de leurs opérations. Il est vrai que le rôle fiscal dans son ensemble aura changé sur la période étudiée mais l'accroissement de chaque contribution fournira encore une mesure relative de la superficie travaillée par un fermier. S'il prend en charge des terres nouvelles, son assiette augmentera plus vite que le taux global; dans le cas contraire, elle suivra le même mouvement.

Il est donc possible de considérer les changements dans les rôles fiscaux comme une approximation de l'évolution de la taille des fermes; utilisons les rôles des années 1740, époque où, au moins près de Paris, la contribution semble proportionnelle à la superficie tenue par un fermier, même si les surfaces elles-mêmes sont rarement mentionnées. Pour un nombre restreint de propriétés, nous avons les contributions fiscales de 1740-1741 et 1783-1789 (49). Si l'on représente graphiquement l'évolution pour chaque propriété et qu'on la compare à celle de la PTF, pour la même époque et la même terre, la relation entre l'échelle de l'activité des fermiers et la PTF ressort clairement, même si la comparaison ne porte que sur 7 propriétés (fig. 3).

Insistons sur le fait que si le taux de la fiscalité globale par hectare s'est modifié entre 1740-1741 et 1783-1789, le changement dans la taxation pour une propriété donnée fournit tout de mếme une mesure relative de l'extension de la superficie cultivée. A Tremblay-lès-Gonesse, par exemple, l'ampleur de l'activité du fermier augmente considérablement entre ces deux dates. Jusqu'en 1741, un tenancier de faible envergure, Mathieu Bignon, cultive les biens de Notre-Dame, en plus des 30 hectares qu'il possède. Mais au début des années 1780 , son successeur André-Paul Hanoteau travaille beaucoup plus de terre. La plus grande taille de l'exploitation entraîne une augmentation sensible de la contribution fiscale du fermier et la PTF de sa ferme avance du même pas, avec un gain de $14 \%$ (50).

Apparemment, la fusion de terres grâce au cumul de baux favorise la productivité. Le fait que nos mesures de la taille des propriétés et du 


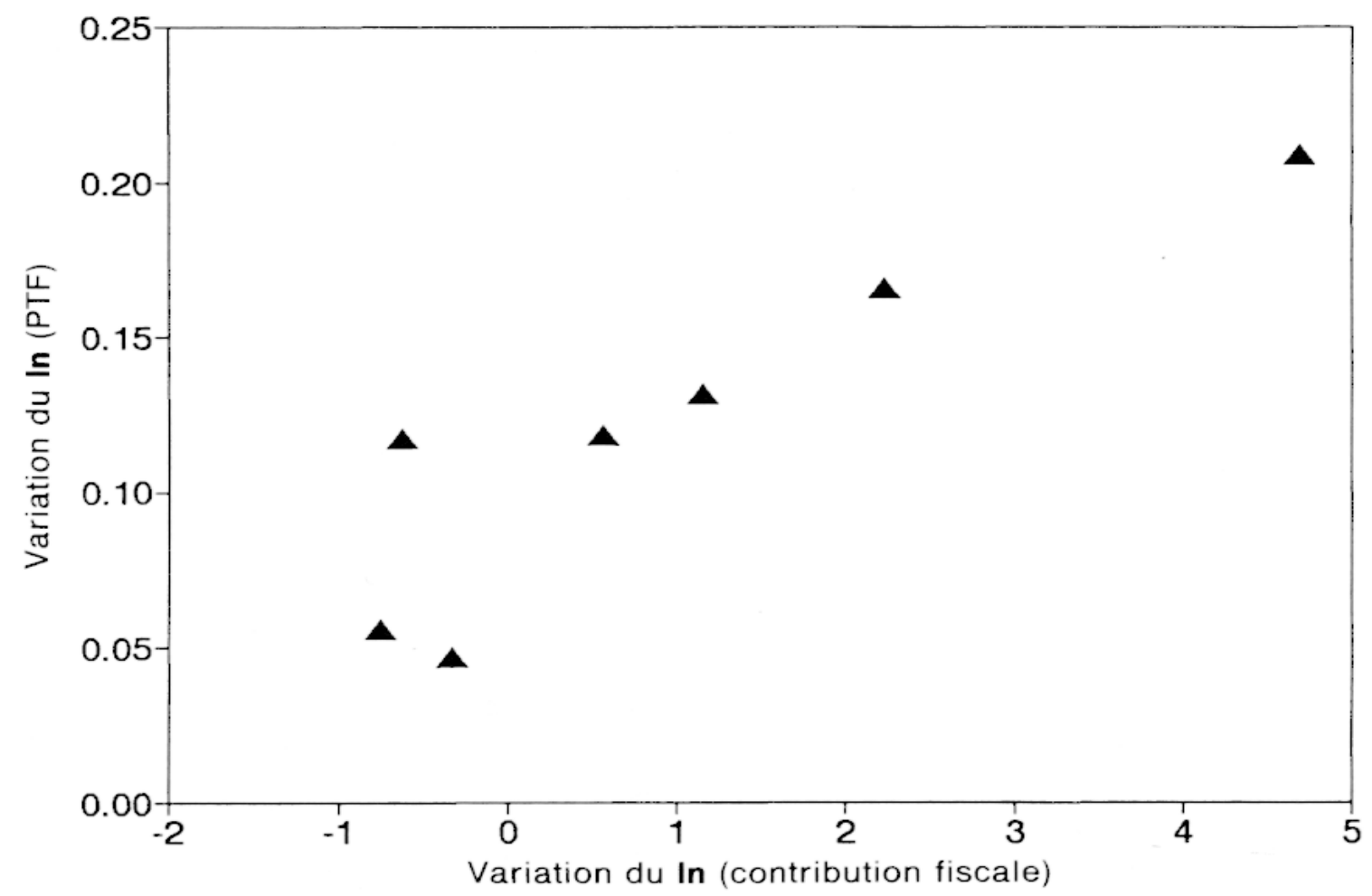

Figure 3 : Variation du In (PTF) et variation du In (contribution fiscale) entre les années $1740-1741$ et les années $1783-1789$ pour 7 propriétés.

regroupement de ces dernières n'ont pas d'effets positifs sensibles sur les régressions du taux de croissance signifie seulement que l'amalgame des propriétés rencontre peu d'obstacles, au moins au XVIII ${ }^{\mathbf{e}}$ siècle. Sinon, les grands ensembles, déjà consolidés auraient profité d'un grand avantage et les coefficients pour la mesure de la taille et du regroupement des propriétés auraient été positifs et élevés dans les régressions de la PTF. Pour opérer sur de plus grandes fermes, les tenanciers regroupent différentes propriétés de telle sorte que la distribution de la taille réelle des exploitations est indépendante de celle de la taille des propriétés. Avec de tels résultats, il est peu probable que cette dernière intervienne de manière significative dans les régressions de la PTF, même s'il y avait des rendements d'échelle croissants.

La taille et le reoroupement importent donc mais, près de Paris au moins, il y a peu d'obstacles pour atteindre la taille approprié. Il est possible que cette échelle ait augmenté au cours du temps, surtout au $\mathrm{XVIII}^{\mathrm{e}}$ siècle. Il est vrai que les tentatives de fusion de propriétés avant cette période ont souvent échoué. Il est également possible que les talents nécessaires pour diriger de grandes fermes soient rares aux époques antérieures, quand bien peu d'individus sont capables de mobiliser les capitaux indispensables et de maintenir les comptes de la ferme. Rien n'entrave le chemin de fermiers ainsi dotés : ni les droits communaux, ni la propriété villageoise ne sont des barrières au regrou- 
pement des terres. Ont-ils cependant gêné ce dernier comme l'ont souvent affirmé les historiens? Il aurait fallu pour cela que les coefficients de la taille et du regroupement des propriétés soient positifs, ce qui n'est pas le cas.

Pondérer les différents facteurs qui soutiennent la PTF est hasardeux mais nous pouvons au moins avancer quelques hypothèses pour le XVIII $^{\mathrm{e}}$ siècle. Entre $1725-1749$ et $1775-1789$, la PTF augmente d'à peu près $9 \%: 1 \%$, peut-être, résulte de l'amélioration des transports, ce qui laisse $8 \%$ pour la croissance de la production agricole relativement à celle des facteurs de production utilisés.

La quantité totale de terre et d'animaux employée ne semble pas beaucoup changer mais les comptes de la ferme analysés par $J$. Postel-Vinay et J.-M. Moriceau suggèrent que le volume de travail diminue d'environ $6 \%$ entre $1725-1749$ et $1775-1789$, sans doute à cause des regroupements de fermes. Ce chiffre se traduirait par un gain de $2 \%$ de la PTF. Quant aux volumes produits, les productions animales selon toute vraisemblance restent stables, mais l'évolution des rendements en grains sont incertains. D'un côté, $\mathbf{J}$. Meuvret et d'autres suggèrent qu'il n'y a pas d'augmentation des rendements près de Paris au XVIII ${ }^{\mathbf{c}}$ siècle et, de là, aucun rôle pour la production des grains dans la croissance de la PTF. D'un autre côté, G. Grantham propose une hausse de $15 \%$ de ces mêmes rendements entre 1750 et 1800 , ce qui équivaut à un gain de $6 \%$ pour la PTF. Ces estimations de Grantham rejoignent celles que d'autres chercheurs ont récemment proposées; si nous les acceptons, elles permettent de rendre compte, avec la baisse du nombre d'heures de travail utilisées, de presque toute la progression de la PTF (51).

Quelles qu'en soient les causes, il est désormais évident que l'agriculture de l'Ancien Régime est capable d'une notable croissance, au moins près de Paris. Cette région est celle où la commercialisation des biens est la plus intense; aucune autre ville n'a pu susciter autant d'occasions favorables au développement. De plus, les innovations qui aiguillonnent la hausse de la productivité - parmi lesquelles le regroupement des terres et l'extension des prairies artificielles - rencontrent moins d'obstacles dans la région parisienne qu'ailleurs. Aussi, les performances de l'agriculture de la région parisienne sont étonnantes. Dès le XVI ${ }^{c}$ siècle, certains fermiers surpassent leurs équivalents anglais et les progrès qu'ils réalisent à la fin du XVIII ${ }^{c}$ siècle sont remarquables, contrairement à ce qui est couramment admis pour la période qui précède la Révolution.

Le problème est que les fermiers du Bassin Parisien ne réussissent pas à maintenir durablement cette croissance de la productivité. Les gains du début du XVI ${ }^{e}$ siècle s'effacent avec les guerres de religion; leur récupération au début du XVII ${ }^{\mathrm{e}}$ siècle est sapée par la fiscalité de la guerre de Trente Ans et par la Fronde. A la longue, l'agriculture souffre gravement de ces reculs. Alors que les agriculteurs des Midlands anglais maintiennent le taux de croissance de leur productivité entre 0,2 et $0,3 \%$ pendant plus de deux siècles, ce taux sur la même période n'est que de $0,1 \%$, peut-être $0,2 \%$ pour la région étudiée. Les fermiers peuvent accélérer ce rythme jusqu'à $0,3 \%$ sur 50 ou 75 ans mais une crise 
exogène bientôt brise leur avance (52). Aussi leur faut-il trois siècles pour accomplir ce que les Anglais réalisent en deux. Leur productivité n'est pas statique ou sans changement; au contraire, elle évolue constamment, marquée par des mouvements de hausse et de baisse. Parmi les forces qui les expliquent, il y a non seulement les opportunités d'échange dans les alentours d'une grande ville mais aussi les conséquences dommageables de la guerre.

Il est un thème qui persiste derrière toutes ces variations, c'est l'influence de la politique. Après tout, c'est la montée de la monarchie absolue qui entretient la montée de Paris et donc, indirectement, celle de la productivité des entrepreneurs de culture des alentours. C'est la fiscalité plus lourde imposée par le roi qui casse la croissance dans les campagnes, ce sont les crises politiques et religieuses qui plongent le royaume dans la guerre civile. Invoquer ce facteur pour expliquer le développement économique peut sembler inapproprié car l'impact des changements politiques semble peu consistant face aux forces de longue durée comme la taille de la population. Peut-être, cependant, serait-il nécessaire de ne plus négliger désormais le rôle du facteur politique dans la croissance économique de longue durée.

Philip T. HOFFMAN

\section{NOTES}

1. L'auteur remercie Robert ALLEN, Philip BENEDICT, Jean-Michel CHEVET, Jack GOLDSTONE, George GRANTHAM, Peter LINDERT, Shawn KANTOR, Ted MARGADANT, Larry NEAL, Kate NORBERG, John NYE, Gilles POSTEL-VINAY, Angela REDISH, Jean-Laurent ROSENTHAL, Ken SOKOLOFF et David WEIR.

2. Pour la seule région parisienne, on compte nombre d'excellentes études utilisant les baux: B. VEYRASSAT-HERREN et E. LE ROY LADURIE, "La rente foncière autour de Paris au XVII ${ }^{\mathrm{e}}$ siècle ". Annales ESC, 23, 1968, pp. 541-55; J.-P. DESAIVE, "A la recherche d'un indicateur de la conjoncture : Baux de Notre-Dame de Paris et de l'abbaye de Montmartre", Les fluctuations du produit de la díme: Conjoncture décimale et domaniale de la fin du Moyen Age au XVIII siècle, éd. J. GOY et E. LE ROY LADURIE, Paris, 1972, pp. 44-57; G. POSTEL-VINAY, La rente foncière dans le capitalisme agricole, Paris, 1974 ; J. JACQUART, " La rente foncière, indice conjoncturel?", Revue historique, 514, 1975, pp. 355-76; et les contributions de G. BEAUR et J.-M. CONSTANT, Prestations paysannes: Dimes, rente foncière et mouvement de la production agricole à l'époque préindustrielle, éd. J. GOY et E. Le ROY LADURIE, 2 vols., Paris, 1982.

3. Les sources incluent l'index des actes capitulaires de la cathédrale de Paris dans AN LL 319-350/351, et les baux originaux dans AN S 123-462. J.-P. DESAIVE fut le premier à utiliser AN LL 319-350/51 comme source pour les baux. J'ai examiné cet index moi-même et j'ai aussi consulté tous les baux originaux correspondants. AN S 123-462 contient aussi des descriptions de propriété, des archives sur l'exploitation des terres et des déclarations faites lors de la saisie des propriétés de Notre-Dame en 1790 qui donnent les caractéristiques des différentes propriétés. Toutes les moyennes sont ici calculées en comptant séparément chaque bail; si nous avions pondéré chaque propriété par sa surface, les résultats auraient peu changé. 
4. G. POSTEL-VINAY, «A la recherche de la révolution économique dans les campagnes (1789-1815)", Revue économique, 40, 1989, pp. 1029-1033: J.-P. BOMPARD, T. MAGNAC et G. POSTEL-VINAY, "Emploi, mobilité et chomage en France au XIX ${ }^{c}$ siècle : Migrations saisonnières entre industrie et agriculture ", Annales $E S C, 45,1990$, pp. 55-76. Pour une tentative de calcul de la productivité du travail sous l'Ancien Régime, voir A. WRIGLEY, "Urban Growth and Agricultural Change: England and the Continent in the Early Modern Period », Journal of Interdisciplinary History, 15, 1985, pp. 683-728.

5. Sur les difficultés de comparer des rendements, voir J. MEUVRET, Le problème des subsistances à l'époque Louis XIV, 3 vols., Paris, 1976-88, vol. 1. pt. 1 (Texte). pp. 207-211. M. MORINEAU, Les faux-semblants d'un démarrage économique : Agriculture et démographie en France au $X V I I I^{\prime}$ siècle, Paris, 1971, contient une comparaison de longue durée des rendements en grains mais ses résultats ont été critiqués par E. LE ROY LADURIE: The French Peasantry, 1450-1660, Berkeley, 1987, p. 430.

6. En 1603, par exemple, les chanoines de Notre-Dame sont en procès parce quils sont incapables de collecter la dîme sur les terres récemment mis en culture et semées avec des navets dans le village de Louvres. En 1713-16, ils perdent la dîme sur des prairies artificielles dans le village de Dampmart au profit du curé local. Sur ces exemples, le type de dime que l'historien utilise (des séries de grandes institutions ecclésiastiques comme Notre-Dame) peut montrer un déclin au moment où les améliorations agricoles sc produisent car (comme c'est souvent le cas) les droits sur les nouvelles récoltes appartiennent au curé. Voir AN LL 327-28, fols 12-17; LL 331, fols 210-50. Pour des exemples d'utilisation des dîmes, voir GOY et LE ROY LADURIE : op. cit. note 2.

7. D.MCCLOSKEY, "The Economics of Enclosure: A Market Analysis ", European Peasants and Their Markets, éd. W.N. PARKER et E.L. JONES, Princeton, 1975. pp. 123-76; R. ALLEN, "The Efficiency and Distributional Consequences of Eighteenth-Century Enclosures », Economic Journal, 92, 1982, pp. 937-53.

8. R.G. CHAMBERS, Applied Production Analysis: A Dual Approach. Cambridge, 1988 , pp. 235-39, donne une définition de la PTF. Certains lecteurs peuvent être contrariés par l'hypothèse des proportions constantes, mais elle est raisonnable pour lagriculture ancienne. Pour une discussion, voir P.T. HOFFMAN, "Land Rents and Agricultural Productivity: The Paris Basin, 1450-1800 », Social Science Working Paper 752, California Institute of Technology, Pasadena, CA. 91125, Etats-Unis (désormais cité : SSWP 752). Ce texte donne les formules mathématiques précises pour la PTF et pour son taux de changement et explique pourquoi une population croissante n'affecte pas son indice.

9. Pour l'aspect technique, voir P. HOFFMAN, $S S W P$ 752, qui explique ćgalement comment sont traités les paiements en nature, les pots de vin, les contre-lettres, les charges et un ensemble de problèmes liés. En général, les paiements en nature sont convertis en monnaie aux prix qui prévalent sur la place où les livraisons sont faites. Avec les propriétés de Notre-Dame, les contre-lettres ne changent pas la rente et une sousestimation de cette dernière ne constitue pas un problème. Pour une discussion de ces questions, voir J. JACQUART, art. cit. note 2 et G. BEAUR, Le murché foncier à la veille de la Révolution, Paris, 1984, pp. 231-246.

10. Pour les détails, voir P. HOFFMAN : SSWP 752. Après une discussion sur l'obtention des proportions des productions et des facteurs de production, ce texte présente plusieurs estimations : il montre que les proportions varient peu entre fermes et au cours du temps et démontre que même si les parts variaient, les résultats seraient peu affectés.

11. J.-M. CHEVET, « Le Marquisat d'Ormesson, 1700-1840 : Essai d'analyse économique ", 2 vols., thèse EHESS, dactyl., 1983, 1: 173-74, 383:2:406-23, 440: $\mathrm{P}$. GOUBERT, Beauvais et le Beauvaisis de 1600 à 1730,2 vols., Paris, 1960, tome 1, p. 182 : G. LEFEBVRE, Les paysans du Nord pendant la Révolution française, Bari, 1959 ,

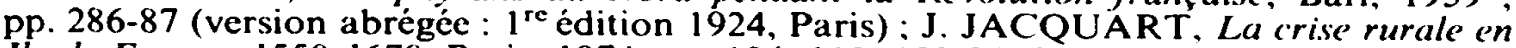
Ile-de-France, 1550-1670, Paris, 1974, pp. 104, 119, 133-34, 352-57, 724, 749-51.

12. P. HOFFMAN, "Social History and Agricultural Productivity: The Paris Bassin, 1450-1800 ", Social Science Working Paper 742. California Institute of Technology (désormais cité SSWP 742), p. 6: J.B. COLLINS. Fiscal Limits of Ahsolutism: Direct Taxation in Early Seventeenth-Century France. Berkeley, 1988. pp. 183-90, 210212 : Idem, "Geographic and Social Mobility in Early Modern France ", texte présenté 


\section{Histoire \& Mesure}

à la Sixteenth Annual Meeting of the Western Society for French History, Los Angeles, 1988 ; M. BAULANT, "Le salaire des ouvriers du bâtiment à Paris de 1400 à 1726 ", Annales ESC, 26, 1971, pp. 463-83; et "Prix et salaires à Paris au XVI" siècle : Sources et résultats ", Annales ESC, 31, 1976, pp. 954-95; A.H. TESSIER et al., Encyclopédie méthodique ou par ordre de matières : Agriculture, 7 vols., Paris, 1787-1821, vol. 1 : articles « affanures », et « avoine »; vol. 3 : articles « faucher » et "faucilleur".

13. M. BAULANT, art. cit. note 12 , pp. 463-83; P. HOFFMAN, SSWP 742, pp. 6-7; J.-P. BOMPARD et al., art. cit. note 4 et Y. DURAND, "Recherches sur les salaires des maçons à Paris au XVIII" siècle ", Revue d'histoire économique et sociale, 44, 1966, pp. 468-80.

14. M. BAULANT, "Prix et salaires à Paris", pp. 980-986 ; G. FOURQUIN, Les campagnes de la région parisienne à la fin du moyen âge, Paris, 1964, p. 496; $\mathrm{P}$. GOUBERT, op. cit. note 11, tome 1, pp. 139-140, 547-60 et J.-P. GUTTON, Domestiques et serviteurs dans la France de l'ancien régime, Paris, 1981, pp. 111-117.

15. M. BAULANT, "Le salaire des ouvriers " et "Prix et salaires à Paris...", pp. 980-986. L'exception majeure concerne les vignerons mais M. BAULANT explique le comportement particulier de leurs salaires par leur situation et par la manière dont ils sont payés.

16. M. BAULANT, «Les salaires des ouvriers à Paris », p. 472 et «Prix et salaires à Paris...", pp. 980-987; M. LACHIVER, La population de Meulan du XVIT au $X I X^{e}$ siècle (vers 1600-1870), Paris, 1969, pp. 91-122; J. BEAUD et G. BOUCHART, "Le dépôt des pauvres de Saint-Denis (1768-1792)", Annales de démographie historique, 1974 , pp. 127-43; P. HOFFMAN, SSWP 752 et le livre à paraître de G. POSTELVINAY et J.-M. MORICEAU.

17. Pour les sources et plus de détails voir P. HOFFMAN, SSWP 752. Tous les coûts du capital sont ici fondés sur le prix du louage du capital, représenté par le prix d'achat du capital, un taux d'intérêt et un taux de dépréciation.

18. J. MEUVRET, op. cit., note 5, vol. 3.

19. AN S 320, 27 novembre 1486 ; S 324A, 25 juin 1781 ; S 359, 22 novembre 1496 ; S 380B ; Dupré de Saint Maur, Essai sur les monnoies ou reflexions sur le rapport entre l'argent et les denrées, Paris, 1746, p. 127 (1644).

20. S.L. KAPLAN, Provisioning Paris : Merchants and Millers in the Grain and Flour Trade during the Eighteenth Century, Ithaca, 1984, pp. 206, 215-16; J. JACQUART, op. cit., note 11 , pp. 764-766; J. DUPÄQUIER et al., Mercuriales du pays de rance et du Vexin français (1640-1792), p. 233 ; M. BAULANT et J. MEUVRET, Prix des céréales extraits de la mercuriale de Paris (1520-1698), 2 vols., Paris, 1960-62, tome 1, pp. 12, 25. Comme nous le verrons, les prix périphériques semblent avoir augmenté par rapport à ceux de Paris entre le XVII ${ }^{c}$ et le XVIII ${ }^{c}$ siècles. Voir M. BAULANT, "Le prix des grains à Paris de 1431 à $1788 »$, Annales ESC, 23, 1968, pp. 520-40.

21. G. LEFEBVRE, op. cit., note 11, pp. 103-106, 274-282 ; G. POSTEL-VINAY, La rente foncière dans le capitalisme agricole, Paris, 1974, pp. 51-52; J. JACQUART, op. cit. note 11, p. 132; Idem, art. cit. note $2 ; \mathrm{J}$. VINCHON, Le livre de raison d'une famille picarde: Les Vinchon (1488-1947), Doullens, 1948, pp. 36-37, 98-103; H. NEVEUX, Vie et déclin d'une structure économique : Les grains du Cambrésis (fin du XIV'-début du XVII" siècle), Paris, 1980, pp. 45-49.

22. Un droit de marché existe parfois au nord de Paris, voir J. VINCHON, op. cit., note 21 , pp. 36-37, 98-103 et G. POSTEL-VINAY, op. cit. note 21 , pp. 44-49. Mais il semble inconnu dans le reste du Bassin Parisien, et même dans le nord de Paris il n'est pas universel ; cf. J. JACQUART, art. cit. note 2, p. 375 . Le droit de marché a sans doute persisté dans les régions les moins commercialisées.

23. AN S 201 (1756), S 402 (23 avril 1766), S 407 (25 août 1785).

24. C. ESTIENNE, L'agriculture et la maison rustique, Paris, 1564, fols 8-9; L'art d'augmenter son bien ou règles générales pour l'administration d'une terre, AmsterdamParis, 1784, pp. 171-75. Notre-Dame semble déloger facilement les mauvais tenanciers pour de meilleurs ; pour un exemple, voir P. HOFFMAN, $S S W P$ 742, p. 11.

25. G. POSTEL-VINAY, op. cit., note 21, pp. 35-54 ; G. Grantham, "Agricultural Supply during the Industrial Revolution : French Evidence and European Implications ", Journal of Economic History, 49 (1989) : pp. 43-72 ; G. BEAUR, op. cit. note 9, Paris, 
1984, pp. 263-264. La région étudiée par G. POSTEL-VINAY connaît le "droit de marché " et les fermiers peuvent y baisser les rentes.

26. Comme c'est fréquent en économie, les régressions sont calculées à partir de logarithmes ce qui permet de discuter les pourcentages de changement de la rente.

27. C. ESTIENNE, op. cit. n. 24, fols 8-9; L'art d'augmenter son bien. op. cit. note 24, pp. 10-17, 171-175; Abbé ROZIER, Cours complet d'agriculture, 10 vols., Paris, 1781-1800, vol. 2, article " bail "; AN LL 337-38, fols. 96-101 (1748), fols. 236-37 (1747): LL 350-51, fols. 122-24 (1761-62); S 242 (1754-62); S 247 (1693); S 176 (1666-69).

28. J.-M. MORICEAU, "Un système de protection sociale efficace : Exemple des vieux fermiers de l'lle-de-France (XVII ${ }^{\circ}$-début XIX siècle)», Annales de démographie historique, 1985, pp. 127-44. Ces tenanciers qui s'attardent sont probablement les meilleurs, retenus par leurs propriétaires.

29. Voir P. HOFFMAN, SSWP 752. On peut se demander si les profits de l'entrepreneur varient assez dans le court terme pour perturber la tendance de la PTF. La raison la plus probable serait que l'inflation augmente les profits avant que la rente n'augmente. Heureusement, on peut tester ces phénomènes et montrer qu'ils ne constituent pas un problème. Voir P. HOFFMAN, "Land Rents and Agricultural Productivity : The Paris Basin, 1450-1789 ", Journal of Economic History, vol. 51, $n^{\circ} 4$, décembre 1991, pp. 771-805.

30. Cet exemple, d'après des données aimablement fournies par G. POSTELVINAY, concerne une ferme très productive au nord de Paris. On peut comparer sá productivité vers 1740 et vers 1780 en utilisant les intrants et les extrants physiques via un indice de la productivité de Törnqvist. Cet indice est équivalent à l'usage d'une fonction de production translog mais il permet de comparer la productivité sans faire de régressions. On trouve ainsi que la productivité de la ferme augmente de $9,79 \%$ entre 1740 et 1780 . Si, au contraire, on utilise la méthode retenue dans ce texte, on obtient presque le même résultat : $9,03 \%$. Les résultats appuyés sur les prix sont donc très près de ceux appuyés sur les quantités. Pour plus de détails, voir P. HOFFMAN, SSWP 752.

31. L'ajustement pour la qualité de la terre s'appuie sur les coefficients de la régression I du tableau 1 pour corriger les variations de la rente quand des propriétés rentrent ou sortent de l'échantillon. L'ajustement prend en compte les changements dans les moyennes de l'échantillon pour les variables suivantes : la qualité du sol, la distance à Paris, la taille de la propriété et la quantité de près et de vignes. Pour une explication détaillée, voir P. Hoffman. SSWP 752. Notons que toutes les proprićtés, petites et grandes, interviennent équitablement dans la figure 2 . Introduire une pondération ne changerait pas notablement le graphique.

32. Puisque PTF $=(\mathbf{r}+\mathbf{t})^{\mathrm{s}} \mathbf{C} / \mathbf{P}$, l'omission de la fiscalité $\mathbf{t}$ tendrait à sous-estimer le niveau et le taux de croissance de la PTF si les impôts augmentent relativement aux rentes. Les impôts t pour chaque propriété ne sont jamais connus avec précision mais une supposition raisonnable est que, pour la i-ème propriété, la fraction de la rente brute (c'est-à-dire, rente plus impôts) qui va au propriétaire plutôt qu'au fisc est $\mathbf{g}$, où $\ln (\mathbf{g})=$ $b \ln \left(t_{n} / r_{a}\right)+c_{i}$. Ici $b$ est une constante négative, $t_{\mathbf{a}}$ est la moyenne per-capita de la contribution fiscale, $r_{a}$ est la moyenne de la rente par hectare et $c_{i}$ est une constante qui varie selon la propriété. Avec cette supposition, nous pouvons estimer la grandeur de l'erreur faite en omettant les impôts de la formule de la PTF. Pour le faire, il faut régresser le taux de croissance de la PTF mesuré sans les impóts sur le taux de croissance de $\mathbf{t}_{\mathbf{a}} / \mathbf{r}_{\mathbf{a}}$ que lon peut tirer des reçus fiscaux, des statistiques de la population et des niveaux moyens de la rente. On soustrait ensuite le produit du taux de croissance de $t_{a} / r_{\mathbf{a}}$ et de son coefficient de régression de la mesure du taux de croissance de la PTF dans le but de corriger le taux de croissance de la PTF pour l'omission des impóts. Pour ajuster le niveau mesuré de la PTF, on soustrait le même coefficient de la régression multiplié par $\ln \left(t_{\mathbf{a}} / \mathbf{r}_{\mathbf{a}}\right)$. Tableau 2, colonne 2 contient la régression nécessaire; lerreur impliquée par la non considération des impôts est faible. Voir P. HOFFMAN, SSWP 752.

33. AN S 407 (1464), S 272 (1522, 1545), S 409 (1479, 1482, 1483, 1498, 1511). Cf. G. FOURQUIN, op.cit. note 14, pp. 389-397, 430-531, carte 5.

34. G. FOURQUIN, op.cit. note 14, pp. 389-397, 430-531: J. JACQUART, op.cit. note 11, pp. 42-47.

35. AN LL 329-30. La Grande Paroisse (1594): S 242 (25 juin 1597): J. JACQUART, op, cit. note 11, pp. 171-207. Le déclin de $25 \%$ de la PTF provient dune régression reportée plus bas. 


\section{Histoire \& Mesure}

36. La régression avec $\ln (r e n t)$ suggère que les prix en hause, plutôt que les rentes en déclin, expliquent la baisse de la PTF car la variable fictive pour les années de guerre 1589-1597 n'a pas un coefficient élevé ou significatif (Tableau 1, régression 1). Le principal argument contre la réalité de cette baisse de la PTF se présenterait comme suit : le siège de Paris en 1589-90 a momentanément augmenté le prix des grains en ville, celui qui figure dans notre indice $\mathbf{P}$ (de là déprimant la PTF), même si le prix à la ferme ct la vraie PFT restent les mêmes. Mais l'indice $P$ est la moyenne des prix pour l'année et pour les 8 années du bail en cours. Il est donc improbable qu'il oscille indûment à cause d'une unique année de crise. Par ailleurs, quelques marchés locaux montrent le même pic des prix en 1589-90 qu'à Paris ce qui suggère que l'accroissement du prix n'est pas confiné à la capitale. Cf. J. JACQUART, op. cit. note 11 , p. 765.

37. J. JACQUART, op.cit. note 11, pp. 623-699.

38. J. MEUVRET, op.cit. note 5, vol. 3, pp. 116-134; M. BAULANT, op. cit. note 20: AN F 11207 (prix de Soissons, corrigés suivant les indications de $P$. GOUBERT, op.cit. note 11 , p. 408) ; J. DUPẤQUIER, op. cit. note 20 . Le graphique des prix de Soissons et de Pontoise montre qu'ils montent de $5 \%$ à $10 \%$ par rapport à ceux de Paris entre 1650 et 1750 , avec un décalage plus fort pour Soissons que pour Pontoise, plus proche.

39. Pour des preuves directes de la baisse du coût des transports, voir J. LETACONNOUX, "Les transports en France au $18^{\circ}$ siècle ", Revue d'histoire moderne et contemporaine, 11, 1908-1909, pp. 97-114, 269-292. Une partie de l'amélioration implique certainement la mise en place d'un réseau d'intermédiaires spécialisés, sujet sur lequel je poursuis des recherches. Séparer transports et agriculture est ici bien sûr artificiel puisque l'essentiel du grain est convoyé au marché par les fermiers eux-mêmes.

40. N.F.R. Crafts, British Economic Growth during the Industrial Revolution, Oxford, 1985, pp. 83-85; R. ALLEN, "The Growth of Labor Productivity in Early Modern English Agriculture ", Explorations in Economic History 25, 1988, p. 117-146.

41. AN S 242, 1754-62 (Dampmart); S 282, 1746-55 et S 460, 1782 (La Grande Paroisse).

42. G. BEAUR, op. cit., note 9, pp. 262-268; BERTRANDY-LACABANE, Brétigny-sur-Orge, pp. 314-315; B. VEYRASSAT-HERREN et E. LE ROY LADURIE, art. cit., note 2 . Les historiens peuvent se demander si la compétition croissante entre les tenanciers ou la comptabilité plus précise des propriétaires permettent à ces derniers d'obtenir plus de leurs fermiers à la fin du XVIII ${ }^{\mathrm{c}}$ siècle, ce qui expliquerait la croissance de la rente que l'on observe. Le problème avec cet argument est qu'il n'y a pas de signe d'une rotation croissante entre tenanciers à cette époque, signe attendu d'une telle argumentation. Par ailleurs, il y a de nombreux exemples de pression sur les fermiers antérieurement à cette période.

43. Une cause d'inquiétude est la valeur élevée dans le tableau 1 de l'indice de singularité, signe de multicollinéarité. Bien que la multicollinéarité puisse donc jeter quelque doute sur les résultats avec In (PTF), elle ne touche pas la régression avec le taux de croissance de la PTF, qui atteint le même bas niveau en 1589-97 et la même brutale croissance après 1775 (Tableau 2, régression 2). Selon les coefficients, le taux de croissance de la PTF tombe de $2,4 \%$ en $1589-97$ et reprend un point après 1775 . Toutes les autres indications fournies par la régression s'adaptent à notre interprétation. Pour les détails techniques, voir: G.G. JUDGE et al., The Theory and Practice of Econometrics, New York, 1985, pp. 896-904.

44. Les deux derniers paragraphes s'inspirent de J-M CHEVET, op. cit. note 11 et G. GRANTHAM, "The Diffusion of the New Husbandry in Northern France, 18151840 ", Journal of Economic History, 38, 1978, pp. 311-337: idem, art. cit. note 25: J. JACQUART, op. cit. note 11 , pp. 321-330; J. MEUVRET, op. cit. note 5 et le livre à paraitre de G. POSTEL-VINAY et J.-M. MORICEAU.

45. Pour une comparaison entre taux de croissance de villes modernes, voir: J. de VRIES, European Urbanization, 1500-1800, Cambridge, Mass. 1984.

46. Hanoteau meurt en 1785 et, selon le rôle de taxe de cette année, il exploite 224 hectares. Les papiers de sa succession suggèrent que cette exploitation est même plus grande $(400 \mathrm{ha})$. Je remercie G. POSTEL-VINAY et J.-M. MORICEAU pour cette information.

47. Au XVIII' ${ }^{\mathrm{c}}$ siècle, Notre-Dame veut supprimer les bâtiments sur les propriétés qui ne sont pas assez grandes pour être des fermes économiques: AN LL 332 (1761-62, 
Larchant); S 320 (26-6-1780, Lizy-sur-Ourcq). Un signe de la plus grande fréquence du cumul des baux au XVIII ${ }^{\mathrm{c}}$ siècle est que les baux commencent à inclure une clause le reconnaissant : AN S 324A (Le-Mesnil-Amelot, 25-6-1781), S 407 (Viercy, 25-8-1785). Pour des regroupements antérieurs, voir J. JACQUART, op. cit. note 1 1, pp. $340-348 \mathrm{ct}$ pour un excellent exemple, voir le livre à paraitre de G. POSTEL-VINAY et J.-M. MORICEAU.

48. AN LL 329-30, S 272, S 273 (1636-54).

49. Pour la nature des rôles de taxe dans l'élection de Paris, voir J. GUEROUT, éd. Róles de la taille de l'élection de Paris conservés aux Archives nationales (sous sériés $Z$ IG) et dans les archives départementales, Paris, 1981. Une recherche aux Archives Nationales a mis au jour 44 contributions fiscales de tenanciers dans les séries $Z 1 G$; elles montrent les relations étroites entre le montant de la contribution et le nombre d'hectares exploités. Pour seulement 7 propriétés, cependant, il a été possible d'avoir des baux et des contributions pour $1740-41$ et $1783-89$. Les 7 propriétés étaient situées à Dampmart, Epiais, Gentilly, Louvres, Roissy, Le-Tremblay-lès-Gonesse et Villiers-sur-Marne. Quand plus d'une contribution était disponible pour une propriété, j’ai calculé la moyenne du logarithme de chacune des contributions.

50. AN Z 1G 291 B (1740), 292B (1741), 431 A (1786) et 451 B (1789). Hanoteau meurt en 1785 , ce qui m'oblige à utiliser la contribution fiscale de sa femme pour 1786 et 1789 ; utiliser sa propre contribution pour 1785 n'aurait pas beaucoup changé les choses. Comme pour toutes les propriétés, le changement de In(PTF) est ici calculé entre 1732-45 et $1777-89$. On a choisi de si longues périodes à cause de la variablilité des rentes et parce que les baux en force dans les années 1740-41 et 1783-89 ont été conclus dès 1732 et 1777.

51. G. POSTEL-VINAY et J.-M. MORICEAU, à paraître; J. MEUVRET, op. cit. note 5, vol. 1, pt. 1, pp. 194-203 ; G. GRANTHAM, "The Growth of Labour Productivity in the 'Cinq Grosses Fermes' of France, 1750-1933 ", in B. CAMPBELL et M. OVERTON, eds., Productivity Change and Agricultural Development, Manchester (à paraître). J.-M. CHEVET, op. cit. note 11, propose même des rendements plus élevés que G. GRANTHAM.

52. Les résultats pour les Midlands proviennent de R. ALLEN, op. cit. note 40. La PTF dans les Midlands augmente peut-être de 30 à $60 \%$ entre 1600 et 1800 . Dans le Bassin Parisien, la même croissance prend un siècle de plus : de 1500 à 1789 . 
TABLEAU 1

Régressions avec LN(Rente) et LN(PTF)

\begin{tabular}{|c|c|c|c|}
\hline $\begin{array}{l}\text { Régression: } \\
\text { Variable dépendante }\end{array}$ & $\begin{array}{c}(1) \\
\operatorname{Ln}(\text { Rente })\end{array}$ & $\begin{array}{c}(2) \\
\operatorname{Ln}(\mathrm{PTF})\end{array}$ & $\begin{array}{c}(3) \\
\operatorname{Ln}(\mathrm{PTF})\end{array}$ \\
\hline \multicolumn{4}{|l|}{ VARIABLES INDÉPENDANTES } \\
\hline Constantes & $\begin{array}{r}-11,23 \\
(-16,69)\end{array}$ & $\begin{array}{l}0,079 \\
(0,42)\end{array}$ & $\begin{array}{c}0,11 \\
(0,60)\end{array}$ \\
\hline $\begin{array}{l}\text { Variable indicatrice : année } 1775 \\
\text { et après }\end{array}$ & $\begin{array}{l}0,075 \\
(0,60)\end{array}$ & $\begin{array}{l}0,064 \\
(1,97)\end{array}$ & $\begin{array}{l}0,056 \\
(1,80)\end{array}$ \\
\hline Variable indicatrice: années de guerre & $-0,097$ & $-0,29$ & $-0,28$ \\
\hline $1589-1597$ & $(0,71)$ & $(-8,08)$ & $(-8,30)$ \\
\hline$\%$ en pré & 0,39 & 0,16 & 0,11 \\
\hline variable indicatrice & $(2,09)$ & $(3,25)$ & $(2,51)$ \\
\hline$\%$ en vignoble & 0,0018 & 0,014 & 0,15 \\
\hline variable indicatrice & $(0,005)$ & $(0,13)$ & $(1,50)$ \\
\hline Variable indicatrice : bonne terre & $\begin{array}{c}0,0050 \\
(0,09)\end{array}$ & $\begin{array}{c}0,00093 \\
(0,06)\end{array}$ & $\begin{array}{r}0,0041 \\
(0,28)\end{array}$ \\
\hline $\begin{array}{l}\text { Ln(Distance à Paris } \\
\text { en } \mathrm{km})\end{array}$ & $\begin{array}{c}-0,27 \\
(-6,24)\end{array}$ & $\begin{array}{l}-0,067 \\
(-5,94)\end{array}$ & $\begin{array}{l}-0,070 \\
(-5,76)\end{array}$ \\
\hline $\begin{array}{l}\text { Variable indicatrice : fermier } \\
\text { reconduit dans son bail }\end{array}$ & $\begin{array}{l}0,021 \\
(0,42)\end{array}$ & $\begin{array}{l}0,019 \\
(1,42)\end{array}$ & $\begin{array}{l}0,026 \\
(2,01)\end{array}$ \\
\hline $\begin{array}{l}\text { Temps (en centaines } \\
\text { d'années) }\end{array}$ & $\begin{array}{c}0,91 \\
(23,11)\end{array}$ & $\begin{array}{l}0,061 \\
(5,49)\end{array}$ & $\begin{array}{l}0,063 \\
(5,82)\end{array}$ \\
\hline Ln surface de la propriété (en hectares) & $\begin{array}{l}-0,085 \\
(-3,42)\end{array}$ & $\begin{array}{l}-0,024 \\
(-3,68)\end{array}$ & $\begin{array}{l}-0,050 \\
(-7,46)\end{array}$ \\
\hline $\begin{array}{l}\mathrm{Ln} \text { (Surface des } \\
\text { parcelles) }\end{array}$ & - & - & $\begin{array}{l}0,043 \\
(6,91)\end{array}$ \\
\hline Observations & 652 & 638 & 581 \\
\hline $\mathrm{R}^{2}$ & 0,57 & 0,31 & 0,37 \\
\hline Ecart type & 0,63 & 0,17 & 0,15 \\
\hline $\begin{array}{l}\text { Moyenne de } \\
\text { la variable dépendante }\end{array}$ & 2,70 & 0,79 & 0,80 \\
\hline Indice de singularité & 87,41 & 93,46 & 92,83 \\
\hline
\end{tabular}

Note: Les années avant 1520 sont omises; les T-statistiques sont entre parenthèses. La PTF est ajustée pour les impôts; pour les détails voir P. HOFFMAN : SSWP 752.

Source: L'échantillon des baux et la description des propriétés. Les sources complémentaires sont décrites dans P. HOFFMAN : SSWP 752. 
Philip T. Hoffman

TABLEAU 2

Régressions avec les taux de croissance de la rente et de la PTF

\begin{tabular}{|c|c|c|c|}
\hline Régression : & (1) & (2) & (3) \\
\hline Variable dépendante & $\begin{array}{c}\text { Taux de croissance } \\
\text { de la rente } \\
(\% \text { par an })\end{array}$ & $\begin{array}{c}\text { Taux de croissance } \\
\text { de la PTF } \\
(\% \text { par an })\end{array}$ & $\begin{array}{c}\text { Taux de croissance } \\
\text { de la PTF } \\
(\% \text { par an })\end{array}$ \\
\hline \multicolumn{4}{|l|}{ VARIABLES INDÉPENDANTES } \\
\hline \multirow[t]{2}{*}{ Constantes } & 1,23 & 0,46 & 0,45 \\
\hline & $(5,78)$ & $(1,32)$ & $(1,09)$ \\
\hline \multirow[t]{2}{*}{ Taux de croissance du ratio prix-coût } & 0,61 & - & - \\
\hline & $(7,26)$ & - & - \\
\hline Taux de croissance des impôts & $-0,44$ & $-4,11$ & $-4,44$ \\
\hline sur la rente & $(-0,11)$ & $(-2,00)$ & $(-2,10)$ \\
\hline Taux de croissance de la & - & 0,24 & 0,23 \\
\hline population parisienne & - & $(6,16)$ & $(5,88)$ \\
\hline Variable indicatrice : année 1775 & - & 1,04 & 1,03 \\
\hline et après & - & $(3,48)$ & $(3,35)$ \\
\hline Variable indicatrice : années de guerre & $-4,14$ & $-2,44$ & $-2,25$ \\
\hline $1589-1597$ & $(-4,85)$ & $(-5,85)$ & $(-5,19)$ \\
\hline \multirow[t]{2}{*}{ Variable indicatrice : réparations } & - & $-0,31$ & $-0,56$ \\
\hline & - & $(-0,85)$ & $(-1,39)$ \\
\hline Variable indicatrice : fermier & $-0,17$ & $-0,085$ & $-0,041$ \\
\hline reconduit son bail & $(-0,58)$ & $(-0,64)$ & $(-0,29)$ \\
\hline LN surface de la propriété (Distance & - & $-0,074$ & $-0,083$ \\
\hline à Paris, en km) & - & $(-0,71)$ & $(-0,70)$ \\
\hline LN(Propriété & - & $-0,024$ & $-0,012$ \\
\hline en hectares) & - & $(-0,43)$ & $(-0,18)$ \\
\hline LN(Surface des & - & - & $-0,022$ \\
\hline parcelles) & - & - & $(-0,34)$ \\
\hline Observations & 648 & 648 & 593 \\
\hline $\mathrm{R}^{2}$ & 0,086 & 0,20 & 0,19 \\
\hline Ecart type & 3,69 & 1,68 & 1,68 \\
\hline Moyenne de la variable dépendante & 1,10 & 0,13 & 0,13 \\
\hline Indice de singularité & 2,57 & 14,34 & 16,63 \\
\hline
\end{tabular}

Note: Les taux de croissance sont égaux au taux de variation des logarithmes calculé bail par bail. Le ratio prix-coût est le ratio des prix agricoles sur les coûts des facteurs de production autres que la terre pour lesquels chaque prix et chaque coût sont pondérés par sa part dans le revenu total. Pour plus de détails, voir HOFFMAN, SSWP 752. Les taux de croissance de la PTF ne sont pas ajustés pour les impôts mais, comme il est montré dans HOFFMAN SSWP 752, l'absence d'ajustement pour la fiscalité n'affecte pas les coefficients de la régression parce que le taux de croissance des impôts relativement à celui des rentes figure parmi les variables explicatives. Les années avant 1520 sont omises; les T-statistiques sont entre parenthèses.

Source: L'échantillon des baux et la description des propriétés. Les sources complémentaires sont décrites dans P. HOFFMAN : SSWP 752. 